\title{
The impact of premature extrauterine exposure on infants' stimulus-evoked brain activity across multiple sensory systems
}

\author{
Gabriela Schmidt Mellado $^{\mathrm{a}, 1}$, Kirubin Pillay ${ }^{\mathrm{a}, 1}$, Eleri Adams ${ }^{\mathrm{b}}$, Ana Alarcon ${ }^{\mathrm{b}, \mathrm{c}}$, \\ Foteini Andritsou ${ }^{\mathrm{a}}$, Maria M. Cobo ${ }^{\mathrm{a}, \mathrm{d}}$, Ria Evans Fry ${ }^{\mathrm{a}}$, Sean Fitzgibbon ${ }^{\mathrm{e}}$, Fiona Moultrie ${ }^{\mathrm{a}}$, \\ Luke Baxter $^{\mathrm{a}, *, 2}$, Rebeccah Slater ${ }^{\mathrm{a}, 2}$

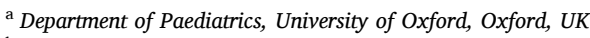 \\ ${ }^{\mathrm{b}}$ Newborn Care Unit, John Radcliffe Hospital, Oxford University Hospitals NHS Foundation Trust, Oxford, UK \\ ${ }^{\mathrm{c}}$ Department of Neonatology, Hospital Sant Joan de Deu, Institut de Recerca Sant Joan de Deu, Universitat de Barcelona, Barcelona, Spain \\ ${ }^{\mathrm{d}}$ Universidad San Francisco de Quito USFQ, Colegio de Ciencias Biologicas y Ambientales, Quito, Ecuador \\ ${ }^{\mathrm{e}}$ Wellcome Centre for Integrative Neuroimaging, FMRIB, Nuffield Department of Clinical Neurosciences, University of Oxford, Oxford, UK
}

\section{A R T I C L E I N F O}

\section{Keywords:}

EEG

Visual

Tactile

Noxious

Auditory

Maturation

\begin{abstract}
A B S T R A C T
Prematurity can result in widespread neurodevelopmental impairment, with the impact of premature extrauterine exposure on brain function detectable in infancy. A range of neurodynamic and haemodynamic functional brain measures have previously been employed to study the neurodevelopmental impact of prematurity, with methodological and analytical heterogeneity across studies obscuring how multiple sensory systems are affected. Here, we outline a standardised template analysis approach to measure evoked response magnitudes for visual, tactile, and noxious stimulation in individual infants $(n=15)$ using EEG. By applying these templates longitudinally to an independent cohort of very preterm infants $(n=10)$, we observe that the evoked response template magnitudes are significantly associated with age-related maturation. Finally, in a cross-sectional study we show that the visual and tactile response template magnitudes differ between a cohort of infants who are agematched at the time of study but who differ according to whether they are born during the very preterm or late preterm period ( $n=10$ and 8 respectively). These findings demonstrate the significant impact of premature extrauterine exposure on brain function and suggest that prematurity can accelerate maturation of the visual and tactile sensory system in infants born very prematurely. This study highlights the value of using a standardised multi-modal evoked-activity analysis approach to assess premature neurodevelopment, and will likely complement resting-state EEG and behavioural assessments in the study of the functional impact of developmental care interventions.
\end{abstract}

\section{Introduction}

Premature birth and early exposure to the extrauterine environment can result in widespread neurodevelopmental impairment, dependent on the degree of prematurity at birth and postnatal insults (Blencowe et al., 2013; Wallois et al., 2020). Relative to in-utero, the extrauterine environment increases exposure to noise, light, handling and clinically painful procedures, as well as pharmacological interventions, which all have the potential to detrimentally influence developmental trajectories
(Pineda et al., 2014; Smith et al., 2011). During the third trimester, dramatic changes to both brain structure and function occur (André et al., 2010; Dubois et al., 2014; Dubois and Dehaene-Lambertz, 2015; Wallois, 2010), and in addition to brain activity being endogenously generated (Colonnese and Khazipov, 2012), neural activity becomes progressively sensory driven in anticipation of patterned environmental sensory input (Colonnese et al., 2010; Milh et al., 2007; Wess et al., 2017). Given the important role of sensory input in neurodevelopment, infants exposed to the extrauterine environment during these critical

\footnotetext{
* Corresponding author at: Paediatric Neuroimaging Group, Department of Paediatrics, Level 2 Children's Hospital, John Radcliffe Hospital, University of Oxford, Oxford, UK.

E-mail addresses: luke.baxter@paediatrics.ox.ac.uk (L. Baxter), rebeccah.slater@paediatrics.ox.ac.uk (R. Slater).

1 These authors contributed equally

2 These authors contributed equally
} 
periods are vulnerable to the influence of developmentally unexpected environmental stimuli.

Prematurity can negatively impact the development of multiple sensory systems (Wallois et al., 2020). Children born prematurely can develop atypical somatosensory profiles (Adams et al., 2015; Wickremasinghe et al., 2013), decreased mechanical and thermal sensitivities related to pain exposure (Walker et al., 2009), sensorineural deafness from excessive noise and drug exposure (McMahon et al., 2012; Zimmerman and Lahav, 2013) and cortical visual impairment (Rosenberg et al., 1996; Van Braeckel and Taylor, 2013). Brain activity evoked by sensory stimulation can be recorded using electroencephalography (EEG) to assess the functional integrity of these developing sensory systems. Somatosensory (Leikos et al., 2020; Majnemer and Rosenblatt, 1996; Vanhatalo et al., 2009), noxious (Hartley et al., 2017; Slater et al., 2010b), auditory (Martins Ribeiro and Mamede Carvallo, 2008; Taylor et al., 1996), and visual (Majnemer and Rosenblatt, 1996; Taylor et al., 1992; Whyte, 1993) evoked potentials have all previously been independently identified in infants using a variety of analytical methods.

However, to date, there is limited data assessing the impact of extrauterine exposure on the developmental trajectory of sensory evoked cortical activity across multiple stimulus modalities within the same infants. This severely limits our understanding of the impact that premature ex-utero development has across these sensory systems in terms of the relative magnitude and directionality of effect. A major challenge to assessing the impact of prematurity across multiple sensory systems is lack of statistical power due to limited sample sizes as well as limitations to the number of stimulus trials that can be applied per infant. At the extreme is assessment of noxious input to study the infant pain system, where sample sizes for infant pain studies are often limited and noxious events are often single-trial clinically-required events, such as heel lances (Courtois et al., 2016).

In this study, we assess the impact of premature extrauterine environmental exposure on the development of evoked EEG brain activity across four stimulus modalities: visual, tactile, noxious, and auditory. We first characterise these stimulus evoked responses in a cohort of healthy term infants. Due to the single-trial nature of clinically required noxious procedures, we uniformly applied a template analysis approach to each stimulus modality, which we have formerly developed and validated for the analysis of noxious-evoked brain activity in neonates (Hartley et al., 2017, 2015). We have previously shown that a template of noxious-evoked brain activity capturing the dominant evoked waveform is an analysis approach robust enough for successful application to single-trial data, sensitive to a range of biologically interesting demographic and clinical variables (Cobo et al., 2021; Green et al., 2019; Gursul et al., 2018; Hartley et al., 2021, 2016; Kasser et al., 2019; Vaart et al., 2019), and provides a sensitive primary outcome in analgesic clinical trials (Hartley et al., 2018).

Here, we systematically apply the template analysis approach to each modality of stimulus-evoked EEG data acquired from a 25-electrode array to optimise and standardise our analysis pipeline and minimise analytic heterogeneity across the stimulus modalities. In an independent cohort of infants born very prematurely, we demonstrate that the stimulus-evoked response templates are sensitive to age-related developmental maturation. Furthermore, we report that the magnitudes of visual- and tactile-evoked responses significantly differ between agematched infants born in the very and late preterm periods, demonstrating the differential impact of premature birth on the maturation of functional sensory responses.

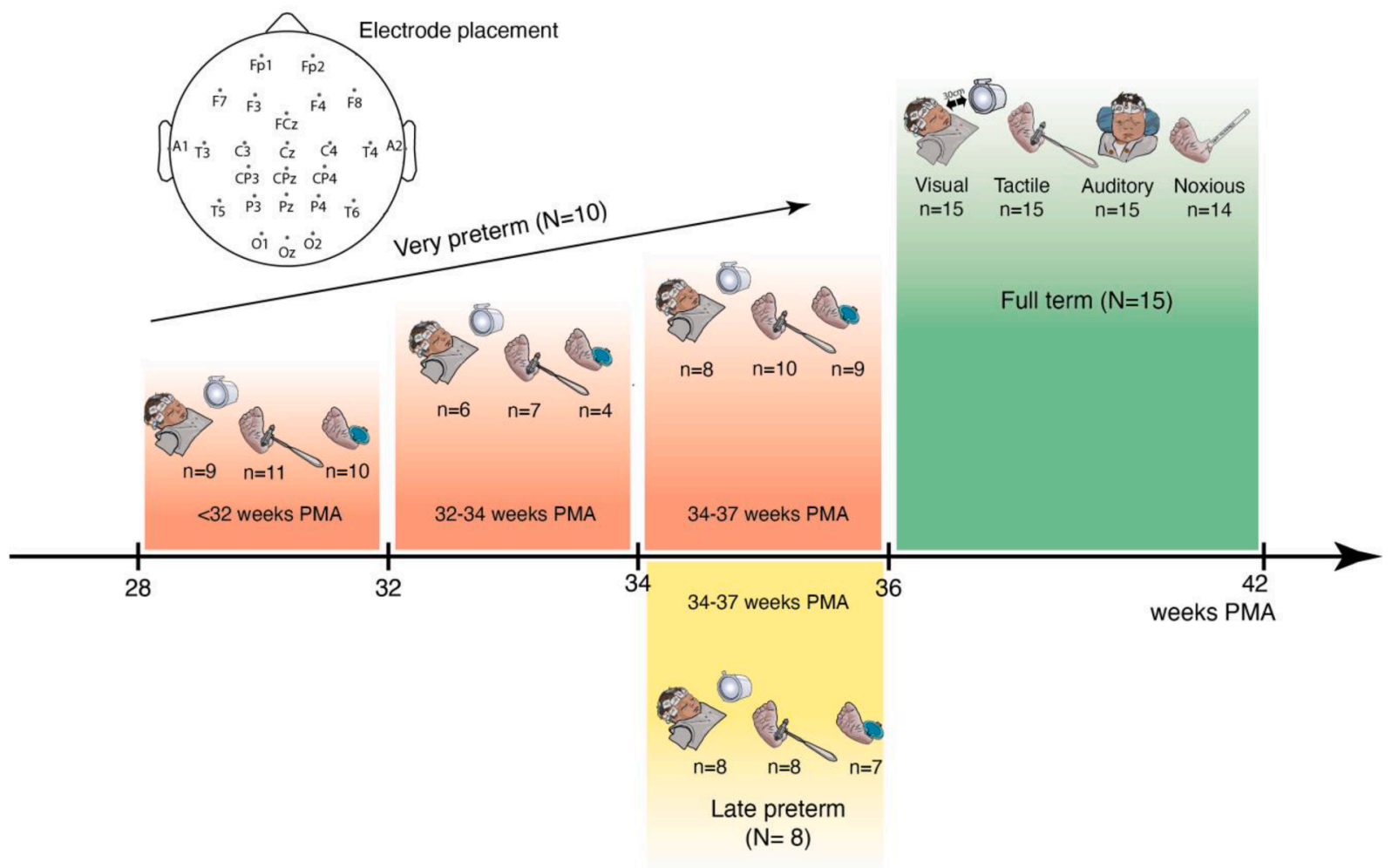

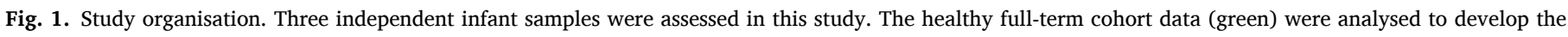

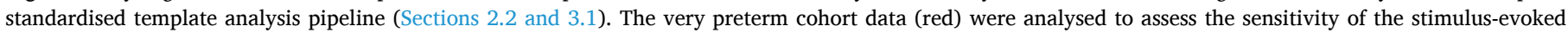

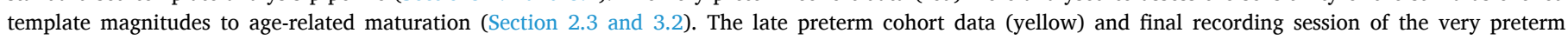

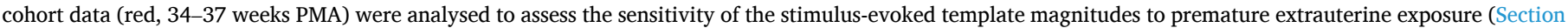

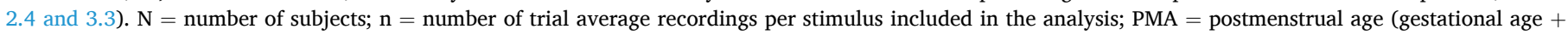

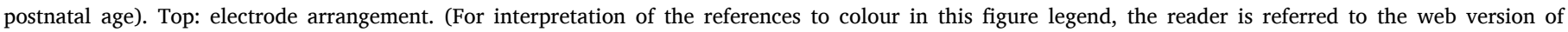
this article.) 


\section{Methods}

\subsection{Overview}

\subsubsection{Study organisation}

This study is divided into three sections. First, EEG data from a cohort of healthy term-born infants (Fig. 1, Full-term) were analysed to establish the template analysis methodology that would be applied across stimulus modalities in subsequent analyses. Second, the outlined template analysis approach was applied to EEG data from an independent cohort of very preterm infants (Fig. 1, Very preterm). These infants were studied longitudinally to assess the association between the stimulus-evoked EEG template magnitude and infants' age at study. Third, the template analysis was applied to EEG data from the very preterm infants' final recording session (Fig. 1, Very preterm, >34 weeks PMA), and these stimulus-evoked EEG template magnitudes were compared to that of an independent cohort of age-matched late preterm infants (Fig. 1, Late preterm). This comparison assessed the association between the stimulus-evoked EEG template magnitude and the degree of extrauterine environmental exposure.

Thus, in this study, the template is derived in the term infants and then applied to independent cohorts of preterm infants. This allows us to scale the unit template magnitude (see Section 2.2.5 below) based on the cohort of term-born infants (who by definition have zero premature extrauterine exposure) and to establish a benchmark against which template magnitudes can be compared in preterm cohorts (who have varying degrees of premature extrauterine exposure).

\subsubsection{Participant recruitment}

Infants born between February 2018 to February 2020 from the Maternity Unit and Special Care Baby Unit at the John Radcliffe Hospital, Oxford, UK, were enrolled in this study after informed, written consent was obtained from the parents. Ethical approval was obtained from an NHS Research Ethics Committee (National Research Ethics Service, REC reference: 12/SC/0447), and research was conducted in accordance with Good Clinical Practice guidelines and the Declaration of Helsinki. Each participant was eligible to take part in the study if they had no major neurological malformations or conditions such as intraventricular haemorrhage (IVH) grade 2 or higher, and no history of maternal substance abuse. At the time of each study, infants were clinically stable, did not require invasive ventilation, and had received no sedatives or analgesics within the prior $24 \mathrm{~h}$. Each cohort is described below, and the full demographic information is detailed in Table 1.

\subsubsection{EEG setup}

All EEG data were recorded with a sampling frequency of $2 \mathrm{kHz}$ using a 25-recording electrode arrangement and CURRY scan7 neuroimaging software suite. Channels included Fp1, Fp2, F3, F4, F7, F8, FCz, T3, T4, C3, C4, Cz, CP3, CP4, CPz, T5, T6, P3, P4, Pz, O1, O2, Oz, A1 and A2 electrodes, with $\mathrm{Fz}$ as reference and $\mathrm{FPz}$ as ground. Electrodes were placed according to the 10-20 International Electrode Measuring System, and recordings acquired by a trained clinical neurophysiologist (GSM).

\subsubsection{Sensory stimulation}

After allowing infants to settle and verifying EEG recording quality,

Table 1

Infant demographics for the three independent cohorts used in this study.

\begin{tabular}{|c|c|c|c|c|c|}
\hline & Full-term & Very preterm & & & Late preterm \\
\hline Number of participants & 15 & 10 & & & 8 \\
\hline Female & 6 & 5 & & & 3 \\
\hline Gestational age at birth (weeks) & $38.2(36.1-41.0)$ & $29.0(27.1-31.4)$ & & & $34.7(34-35.7)$ \\
\hline Birthweight $(\mathrm{g})$ & $3166(2130-4590)$ & $1173(760-1900)$ & & & $2449(1550-4525)$ \\
\hline \multicolumn{6}{|l|}{ Antenatal factors } \\
\hline Prolonged rupture of membranes & 1 & 5 & & & 2 \\
\hline Intrauterine growth restriction (IUGR) ${ }^{1}$ & 1 & 3 & & & 2 \\
\hline Abnormal Doppler & 0 & 1 & & & 1 \\
\hline Maternal infection (Chorioamnionitis) & 0 & 4 & & & 0 \\
\hline \multicolumn{6}{|l|}{ Delivery } \\
\hline Vaginal (assisted/unassisted) & 8 & 2 & & & 5 \\
\hline Caesarean & 7 & 8 & & & 3 \\
\hline Apgar score at 10 mins & 10 & $9(8-10)$ & & & $9.75(8-10)$ \\
\hline \multicolumn{6}{|l|}{ Conditions during admission in NICU } \\
\hline Neurological conditions & 0 & $1 *$ & & & 0 \\
\hline Cardiac conditions & 0 & $1 * *$ & & & 0 \\
\hline Respiratory distress syndrome & 1 & 7 & & & 1 \\
\hline Invasive mechanical ventilation $<24 \mathrm{hrs}$ & 0 & 2 & & & 3 \\
\hline Invasive mechanical ventilation $>24 \mathrm{hrs}$ & 0 & $2 * * *$ & & & 0 \\
\hline Infection suspected & 1 & 4 & & & 2 \\
\hline Previous surgery & 0 & 0 & & & 0 \\
\hline Test occasion & & $<32$ & $32-34$ & $34-37$ & \\
\hline Postmenstrual age at study (weeks) & $38.5(36.6-41.7)$ & $30.4(28-31.9)$ & $32.7(32-33.3)$ & $35.1(34.3-36.3)$ & $35.5(34.7-36.4)$ \\
\hline Postnatal age at study (days) & $3.7(1-7)$ & $9.8(5-15)$ & $23.6(15-36)$ & $44(36-58)$ & $5.9(4-10)$ \\
\hline Respiratory support (LFT/HFT) at study & 0 & 5 & 5 & 4 & 0 \\
\hline Non-standard drugs ${ }^{2}$ at study - Budesonide & 0 & 0 & 1 & 1 & 0 \\
\hline Analgesics $<24$ hrs prior to study & 0 & 0 & 0 & 0 & 0 \\
\hline Antibiotics at study & 1 & 1 & 0 & 0 & 0 \\
\hline Prior pain & $2(0-23)$ & $21.3(11-46)$ & $33.9(22-59)$ & $37.5(25-65)$ & $10(3-21)$ \\
\hline
\end{tabular}

* Choroid plexus cyst.

** Patent ductus arteriosus (PDA).

$* * *$ Infants were ventilated for a maximum of 4 days and did not require further periods of ventilation.

LFT $=$ Oxygen therapy.

HFT $=$ High flow nasal cannulae.

Prior pain $=$ number of skin breaking procedures and endotracheal tube/oral suction.

For age, birthweight, and prior pain, values are presented as means (accumulative mean for prior pain) with range in parentheses.

${ }^{1}$ IUGR is defined as any baby whose weight is below the 10th percentile for their gestational age.

${ }^{2}$ Standard drugs include: Caffeine citrate, Vitamins, Probiotics, Breast milk fortifier, Iron, Sodium and Phosphate supplements. 
each stimulus was applied in a consecutive train of a maximum of 30 trials, allowing at least $10 \mathrm{~s}$ between trials. The visual, tactile, auditory and noxious experimental stimuli were each applied in a randomised order. The clinical procedure (i.e. the heel lance) was always performed first or last depending on clinical need. On some occasions it was not possible to administer all the stimuli if the infant became behaviourally unsettled or minor equipment faults occurred. The precise numbers of stimuli applied to each infant is summarised in Fig. 1. Each test occasion lasted between 20 and 40 mins and commenced when the infant was behaviourally settled. It was not possible to control infants' vigilance states throughout the studies, which is a source of noise variance within the data.

The visual stimulus was a flash of light presented using a Lifelines Photic Stimulator (intensity level 4, approximately $514 \mathrm{~lm}$ ), which was manually held $20-30 \mathrm{~cm}$ from the infant's eyes. Light flashes automatically triggered event marking of the EEG recording. The tactile stimulus was a modified tendon hammer with a $154 \mathrm{~mm}$ diameter contact surface applied gently to the infant's heel. The modified tendon hammer includes a built-in force transducer, which triggered an event mark on the EEG monitor after a fixed minimum intensity is applied (Worley et al., 2012). To ensure a constant stimulus intensity, the weight of the hammer is allowed to gently drop from a fixed angle (the researcher does not exert the force), and the same researcher applied all tactile stimuli throughout.

Experimental or clinical noxious stimuli were applied depending on the cohort of infants and clinical procedures required. The two preterm cohorts (Fig. 1, Very preterm and Late preterm) had a clinical noxious stimulus in the form of a heel lance (BD Quikheel Preemie Lancet Becton, Dickinson and Company) as they clinically required blood sampling at the time of study. The heel lance pierces the skin at a depth of $0.85 \mathrm{~mm}$, and no lance was applied solely for the purpose of this study. The heel lance was only applied once during each recording resulting in a single trial per recording. In the healthy full-term cohort (Fig. 1, Full-term), no infants required a clinical noxious stimulus, so a mild $128 \mathrm{mN}$ experimental sharp-touch stimulus (PinPrick Stimulator, MRC Systems) was applied to the heel of the foot instead (20-30 trials). The pinprick stimulus produces EEG responses of lower amplitude than those of clinical procedures, such as the heel lance, and does not cause behavioural distress (Hartley et al., 2015) but activates nociceptors in the periphery (Magerl et al., 2001) and elicits noxious-evoked brain activity (Baxter et al., 2021; Iannetti et al., 2013; Slater et al., 2010b). Both the experimental pinprick and clinical heel lance stimuli had builtin interfaces to trigger an EEG event mark.

The auditory stimulus was a fixed tone at $1,000 \mathrm{~Hz}$ frequency, delivered using a pair of speakers ( $n=5$ late preterm cohort, $n=3$ very preterm cohort) or by headphones placed firmly on the infant's ears ( $\mathrm{n}$ $=3$ late preterm cohort, $\mathrm{n}=5$ very preterm cohort). The speakers (Xmini MAX II Portable Speakers, Xmi) were placed $15 \mathrm{~cm}$ from the ears and delivered the tone with $100 \mathrm{~ms}$ duration at a volume of $80 \mathrm{~dB}$. The headphones delivered the tone with $4 \mathrm{~ms}$ duration and a volume of 70 $\mathrm{dB}$ nHL using the Surpass Ltd (EMS Biomed) auditory system. Headphones were used for all the infants in the full-term cohort. Technical issues with the initial auditory equipment arose during the study which required the upgrade from speakers to a headphone setup. This change to the stimulus delivery method likely introduced significant noise into the data and reduced the quality of the data recorded in response to the auditory stimuli. For completeness, due to the inclusion of the auditory stimulation in the study design, we included the data acquired following presentation of the auditory stimulus in the initial presentation of the results.

\subsubsection{EEG data preprocessing}

EEG activity evoked by each individual stimulus trial was epoched in the period $\pm 5 \mathrm{~s}$ either side of the stimulus and temporally filtered using 0.5-70 Hz bandpass filter (comprising a high-order low-pass FIR and a second-order high-pass Butterworth filter) as well as a $50 \mathrm{~Hz}$ notch filter.
An average reference derivation was used so that a consistent referencing approach could be applied across the different stimulus modalities. As the topographical distribution of the evoked activity differs between stimulus modalities, use of an average reference reduces the spatial bias that would arise if a sole reference electrode had been selected per stimulus modality. Electrodes in positions more likely to be affected by biological artefacts such as eye blinks (Fp1, Fp2), muscle and ECG artefact (A1, A2), and those which were not part of the standard 10-20 electrode positions (FCz, CP3, CPz, CP4) were excluded when determining the average.

All trials were visually assessed for quality by a trained clinical neurophysiologist (GSM). Trials were rejected only if the pre-stimulus baseline or the post-stimulus activity contained gross movement artefacts (e.g. signal amplitudes $>800 \mu \mathrm{V}$ on any channel), or if the prestimulus baseline was unsettled (i.e. when stimulation was preceded by spontaneous bursting or other activity in the background or when EEG activity prior to stimulus was fluctuating $>20 \mu \mathrm{V}$ ). Of 2,954 trials, 1,874 (visual $=761$, tactile $=903$, pinprick $=210$ ) were retained. An average of 24 trials (range 14-30 trials) were performed per stimulus and a mean of 9 (range 2-20 trials) rejected from a single recording. A single trial heel lance was recorded in all the infants from the very preterm ( 28 recordings) and late preterm (8 recordings) cohorts. In total, 6 heel lance trials out of 36 were rejected due to artefact.

The average of the retained trials (with the exception of the singletrial heel lance stimulus) was used to produce a single stimulusevoked response per infant recording and stimulus. Spherical interpolation was performed on the trial-averages to correct any channels that contained remaining artefacts, largely due to involuntary blinking when the visual stimulus was applied. In each case, a maximum of four channels (not adjacent to each other) were interpolated per recording and a Laplacian arrangement (whereby each channel was considered adjacent to up to four neighbouring channels according to position on the head) was used to determine which electrodes were considered adjacent to one another. All further analyses were done using the trial average per infant and stimulus, which was additionally baselinecorrected using the pre-stimulus mean in the $500 \mathrm{~ms}$ immediately before application of the stimulus.

\subsection{Developing a standardised template analysis approach}

\subsubsection{Participant details}

A standardised template analysis approach was developed using the full-term infant dataset (Fig. 1, Full-term; $\mathrm{n}=15$; mean GA at birth $=$ 38.2 weeks; mean PMA at study $=38.5$ weeks). Each individual was studied on a single occasion within the first 15 days from birth. See Table 1 for full demographic details.

\subsubsection{Electrode selection using functional neuroanatomical considerations and raw amplitude analysis}

To evaluate the brain region or electrode position where maximal amplitude change occurs after application of a stimulus, the evolution of the spatial distribution of amplitude changes immediately after the stimulus was visually assessed. This was done using a grand average of the trial averages per infant for each stimulus. Using a priori functional neuroanatomical considerations, large evoked responses were expected around $\mathrm{Oz}$ for visual stimulation, $\mathrm{Cz}$ for tactile stimulation of the foot, and T3 and T4 for auditory stimulation (Vanderah and Gould, 2020); and from previous publications on EEG activity evoked by the specific noxious stimuli used in the present study, large evoked responses were expected around $\mathrm{Cz}$ (Hartley et al., 2017; Slater et al., 2010b). The preprocessed EEG amplitude changes were visually evaluated to assess these prior expectations and the qualitative biophysical interpretability of the recorded data. If the evoked raw amplitude data were consistent with these prior expectations, these functional neuroanatomical considerations and previous publications formed the basis for electrode selection of a single electrode. 


\subsubsection{Time window selection using temporal cluster analysis}

Having selected the electrode where maximum response amplitude occurred for each stimulus modality, we identified the time window during which the evoked deflections were significantly greater than the pre-stimulus background activity. Using the trial- and subject-averaged timeseries, a time window within the first $800 \mathrm{~ms}$ post-stimulation containing a single positive or negative deflection was identified using non-parametric temporal cluster analysis (Maris and Oostenveld, 2007). Prior to the temporal cluster analysis, all trial-averages were additionally baseline-normalised (dividing by the pre-stimulus standard deviation $500 \mathrm{~ms}$ immediately before the stimulus) in order to minimize discrepancies in the trial-averages due to age-related variance in signal amplitude.

For the temporal cluster analysis, each timepoint in the post-stimulus period was compared to the pre-stimulus period of resting activity across all infants' trial-averaged EEG based on a paired $t$-test at the specific channel of interest. All timepoints exceeding the t-statistic threshold of $97.5 \%$ were considered significant and time-adjacent values were grouped into clusters. The cluster masses (sum of the t-statistics within each cluster) were compared against a permutation distribution of cluster masses derived after repeating this procedure with the poststimulus and pre-stimulus labels randomly re-assigned each time for 1,000 iterations. Remaining clusters whose mass exceeded the permutation distribution in a two-tailed test $(\mathrm{p}<0.025)$ were considered significant. The significant post-stimulation time window was defined as the time range of the first and last timepoint in the significant cluster.

\subsubsection{Waveform selection using PCA and cross-correlation analysis}

We used principal component analysis (PCA) to derive the waveforms that characterised the dominant post-stimulus activity for each stimulus within the stimulus-specific time windows identified using the temporal cluster analysis (Section 2.2.3). For each stimulus modality, the EEG periods across all channels within the specified windows were concatenated together before PCA, and the first three components were considered. We used singular value decomposition (SVD) to project each component onto the data and scale each component waveform for best fit. The scaling factor is the component magnitude. When fitting the component waveforms using SVD, latency differences between individuals were corrected for using Woody filtering (Woody, 1967), which jitters the signal relative to the component to achieve maximum cross-correlation. A maximum jitter of $\pm 50 \mathrm{~ms}$ was selected for visual and noxious stimuli and $\pm 100 \mathrm{~ms}$ for the tactile stimulus (no significant time windows were identified for auditory stimuli). For each component, the cross-correlation between the fitted component waveform and the evoked data was calculated, and the component waveform with the largest cross-correlation within a stimulus modality was selected as the stimulus-evoked response template.

We generated surface heat maps to qualitatively assess the spatial topography of each template's magnitude by fitting the templates to all electrodes. The Woody filtering jitter estimated at each stimulus modalities dominant electrode (identified in Section 2.2.2) was used across all channels. An additional background correction was applied independently for each channel to reduce the effects on the template's magnitudes brought about by changes in the underlying background activity of the signal (such as variations in sleep state) between stimuli and individuals. After estimating the template magnitude in the poststimulus period, the same procedure was applied to successive windows of stable pre-stimulus baseline activity (with length matching the template duration) across the $1 \mathrm{~s}$ period prior to the stimulus. The median of these values resulted in a robust pre-stimulus template magnitude as a surrogate for the background response magnitude and was then subtracted from the stimulus-evoked response template magnitude to obtain a corrected value.

2.2.5. Applying the standardised template analysis approach to novel data Each stimulus-evoked response template was rescaled such that the average template magnitude for the full term cohort used to develop the template was a unit vector i.e. a template magnitude equal to one is the expected value for full term healthy infants. The stimulus-evoked response templates selected in Section 2.2.4 were fit to new stimulusevoked EEG data using the electrode selected in Section 2.2.2 and the time window selected in Section 2.2.3. The fit was performed using SVD to efficiently perform a least squares optimisation, and maximum Woody filtering jitters of $\pm 50 \mathrm{~ms}$ for visual and noxious stimuli and \pm $100 \mathrm{~ms}$ for tactile stimuli. The template magnitudes were corrected using the median pre-stimulus template magnitude, as described in Section 2.2.4.

\subsection{Detecting age-related developmental changes in stimulus-evoked brain activity using the standardised template analysis approach}

\subsubsection{Participant details}

Assessment of the correlation between stimulus-evoked template magnitudes and infant age was performed using the longitudinally studied very preterm dataset (Fig. 1, Very preterm; $\mathrm{n}=10$; mean GA at birth $=29$ weeks). Infants were studied on average three times before discharge from hospital (range 2-4 test occasions). The recordings were later separated into three age groups: $<32$ weeks (mean PMA at study $=$ 30.4 weeks), 32-34 weeks (mean PMA at study $=32.7$ weeks), 34-37 weeks (mean PMA at study $=35.1$ weeks). See Table 1 for full demographic details.

The infants in this cohort were remarkably healthy preterm infants given their young age at birth. A single infant had a minor neuroimaging finding on their ultrasound scan (choroid plexus cyst) of no pathological significance, and that same infant also had a minor cardiac condition. Four infants received mechanical ventilation during their first week of extrauterine life for a short period of time, but not at the time of study. The incidence of chronic lung disease could not be assessed when the last test occasion was performed (infants were too young). The EEG was reviewed by a trained clinical physiologist (GSM). The EEG was classified as dysmature if developmental features such as the incidence of delta brushes, premature temporal theta, discontinuity, and amplitude were not appropriate for postmenstrual age. One infant had a dysmature background EEG at the first test occasion that improved in the subsequent test occasions. The initial abnormal recording was therefore excluded from the analyses. The first test occasion was performed within 15 days of birth and repeated once every 2 weeks thereafter until near discharge from hospital, resulting in 28 recordings. Each study was arranged to coincide with a clinically required heel lance, and no treatments (i.e. phototherapy, humidity protocols) or routine cares were disrupted by the studies.

\subsubsection{Analysis}

The template analysis approach was applied to all infants' stimulusevoked EEG data, as outlined in Section 2.2.5. We used a linear random effects regression model to correlate the stimulus-evoked template magnitudes with infant PMA. Random intercepts were introduced to group repeated recordings from the same patient. GA and the number of trials in each recording's trial average were also separately correlated with the PMA to assess if they were potential confound variables, and no significant correlations were observed for each stimulus.

\subsection{Detecting the impact of premature extrauterine development on stimulus-evoked brain activity using the standardised template analysis approach}

\subsubsection{Participant details}

The impact of premature extrauterine exposure on stimulus-evoked template magnitudes was assessed by comparing responses between very preterm and late preterm infants, both groups matched for age at time of study. The very preterm group was a cohort of infants born $<32$ weeks GA and studied at 34-37 weeks PMA $(n=10$; mean GA at birth $=$ 
29 weeks; mean PMA at study $=35.1$ weeks; mean postnatal age at study $=44$ days). This sample is the same group analysed in the longitudinal study above (see Section 2.3.1 and Fig. 1, Very preterm, $>34$ weeks). See Table 1 for full demographic details.

The late preterm group was a cohort of infants born at 34-36 weeks GA and studied at 34-37 weeks PMA $(\mathrm{n}=8$; mean GA at birth $=34.7$ weeks; mean PMA at study $=35.5$ weeks; mean postnatal age at study $=$ 5.9 days). This sample is an independent sample not used in any previous analyses. Infants had no known neurological or cardiac conditions at the time of study and mechanical support, when given, was not for a prolonged period of time. See Table 1 for full demographic details.

\subsubsection{Analysis}

The template analysis approach was applied to all infants' stimulusevoked EEG data, as outlined in Section 2.2.5. We compared the stimulus-evoked template magnitudes between the PMA-matched very preterm and late preterm infants using a linear random effects regression model. Random intercepts were applied to account for repeated measures in the case of multiple recordings from individuals within a group. Group differences in PMA and the number of trials in each recording's trial average were also separately assessed as potential confound variables, and no significant correlations were observed for each stimulus.

\section{Results}

3.1. A standardised template analysis approach for extracting EEG stimulus-evoked response features across stimulus modalities in infants

\subsubsection{Electrode selection using raw amplitude analysis}

In a cohort of full-term healthy infants $(\mathrm{n}=15$; mean GA at birth $=$ 38.2 weeks, mean PMA at study $=38.5$ weeks), we applied 20-30 trials of visual, tactile, noxious, and auditory stimuli and recorded the stimulus-evoked brain activity using EEG with 25 recording electrodes. To identify the electrodes recording the strongest stimulus-evoked responses, we generated trial- and subject-averaged activity per electrode (Fig. 2). For each modality, large response amplitudes were observed over cortical regions functionally-relevant to the stimulus type. A large evoked response was localised around the midline occipital electrode, Oz, after visual stimulation (Fig. 2 row 1); around the midline central electrode, $\mathrm{Cz}$, after both tactile and noxious stimulation (Fig. 2 row 2-3); and bilaterally around temporal electrodes $\mathrm{T} 3$ and $\mathrm{T} 4$ after auditory stimulation, with larger amplitudes over the left temporal area, T3 (Fig. 2 row 4).

We assessed the stimulus-evoked EEG response amplitudes in successive $200 \mathrm{~ms}$ intervals to demonstrate the spatial evolution of the raw amplitude evoked activity immediately post-stimulus. The visualevoked activity at electrode $\mathrm{Oz}$ featured a positive deflection within the first $200 \mathrm{~ms}$, which evolved into a negative deflection by $400 \mathrm{~ms}$. The tactile-evoked activity at electrode $\mathrm{Cz}$ featured a negative deflection within the first $200 \mathrm{~ms}$, which evolved into a smaller amplitude positive deflection by $400 \mathrm{~ms}$. The noxious-evoked activity at electrode $\mathrm{Cz}$ featured a modest negative deflection within the first $200 \mathrm{~ms}$, which

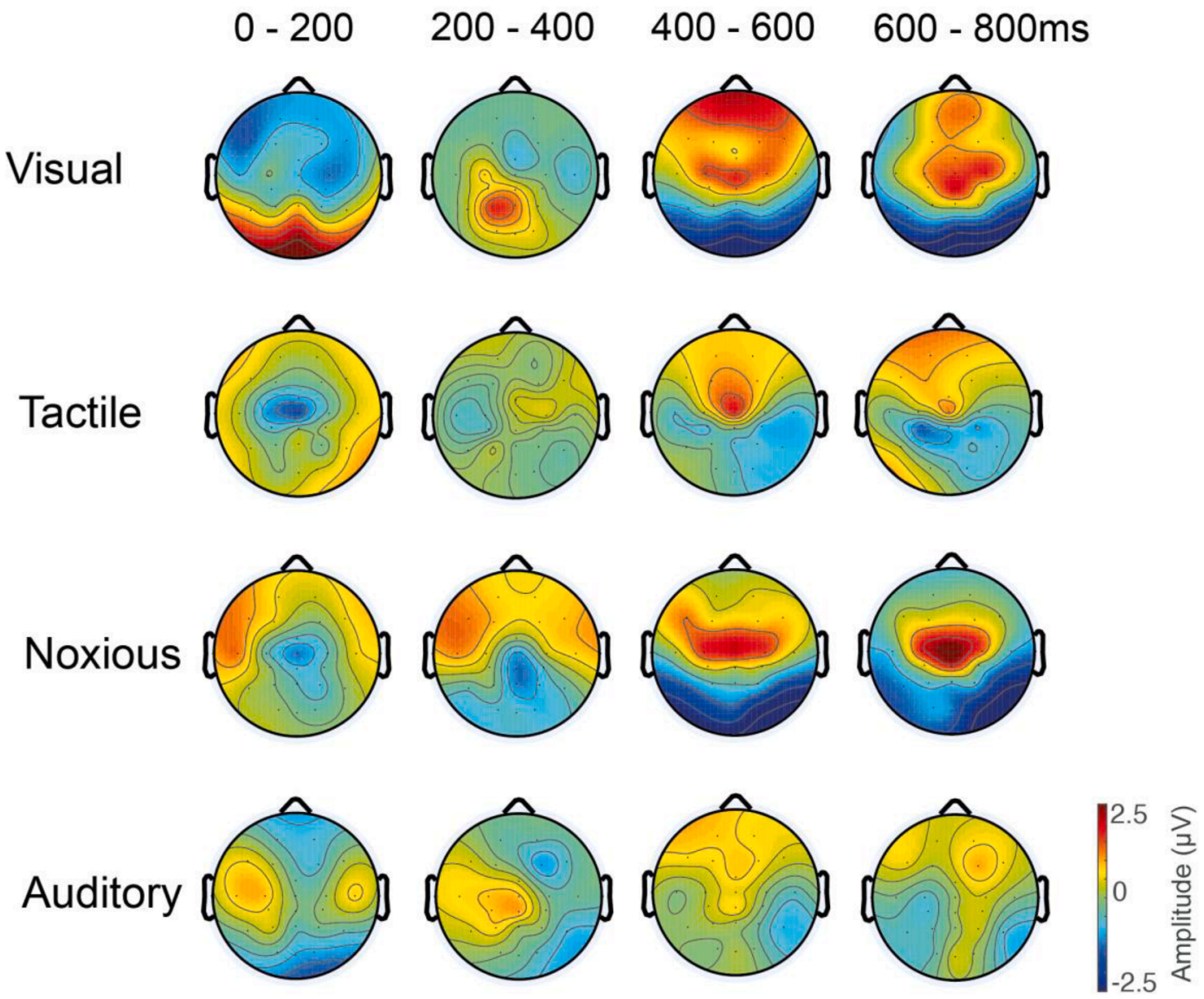

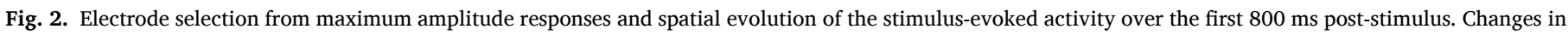

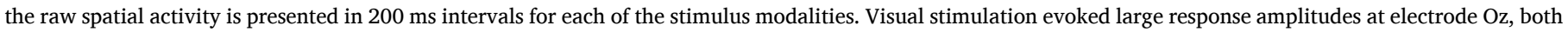
tactile and noxious stimulation evoked large response amplitudes at electrode $\mathrm{Cz}$, and auditory stimulation evoked mild response amplitudes at electrode T3. 
evolved into a larger positive deflection by $400 \mathrm{~ms}$. And the auditoryevoked activity at electrode $\mathrm{T} 3$ featured a modest positive deflection within the first $200 \mathrm{~ms}$.

\subsubsection{Time window selection using temporal cluster analysis}

Having identified the maximum response amplitude electrode for each stimulus modality, we next identified the time window during which these evoked deflections were significantly greater than prestimulus background activity. Using a cluster analysis on the trial- and subject-averaged timeseries, a time window within the first $800 \mathrm{~ms}$ poststimulation was identified, containing a single positive or negative deflection, for the visual, tactile, and noxious stimulus modalities (Fig. 3). No statistically significant time window was identified for the auditory-evoked response. This was very likely due to technical issues related to the presentation of auditory stimulus, as described in the Methods.

For visual-evoked activity at electrode $\mathrm{Oz}$, the $0-231 \mathrm{~ms}$ time window contained a significant positive deflection $(\mathrm{p}=0.001)$. For tactileevoked activity at electrode $\mathrm{Cz}$, the $76-218 \mathrm{~ms}$ time window contained a significant negative deflection $(\mathrm{p}=0.015)$. And for noxious-evoked activity at electrode $\mathrm{Cz}$, the $426-800 \mathrm{~ms}$ time window contained a significant positive deflection ( $\mathrm{p}=0.001$ ).

\subsubsection{Waveform selection using PCA and cross-correlation analysis}

Using the temporal windows containing statistically significant stimulus-evoked responses, we extracted waveforms explaining high proportions of signal variance using principal component analysis (PCA). The dominant waveform morphology per stimulus was then identified as the component with the greatest cross-correlation with the evoked activity. For all three stimulus modalities, the second component (PC2) had the greatest cross-correlation with the average EEG waveform (Fig. 4). For visual-evoked activity, the first three components explained $95.7 \%$ of the variance with scaled cross-correlation values of 19.6, 21.5, and 16.8. For tactile-evoked activity, the first three components explained $87.5 \%$ of the variance with scaled cross-correlation values of $2.94,2.99$, and 1.67. And for noxious-evoked activity, the first three components explained $88.8 \%$ of the variance with scaled crosscorrelation values of 43.1, 59.8, and 42.9.

To assess the biophysical interpretability of the selected PC2 waveforms, the three components for each stimulus modality were reprojected onto the data (Fig. 4). To incorporate the full temporal morphology of the waveforms and improve generalisability to novel datasets, the temporal window in which the components were fit were widened: the visual temporal window was widened from 0 to $231 \mathrm{~ms}$ to 0-250 ms; the tactile temporal window was widened from 76 to $218 \mathrm{~ms}$ to $50-300 \mathrm{~ms}$; and the noxious temporal window was widened from 426 to $800 \mathrm{~ms}$ to $400-800 \mathrm{~ms}$. Goodness of fit between the component's waveforms and the average EEG activity timeseries were assessed visually, and consistent with the quantitative cross-correlation results, PC2 qualitatively captured the dominant evoked deflection with highest fidelity. The visual template fit a positive deflection with a mean template peak latency of $205 \mathrm{~ms}$, the noxious template fit a positive deflection with a mean template peak latency of $576 \mathrm{~ms}$, and the tactile template fit a negative deflection with a mean template peak latency of $181 \mathrm{~ms}$.
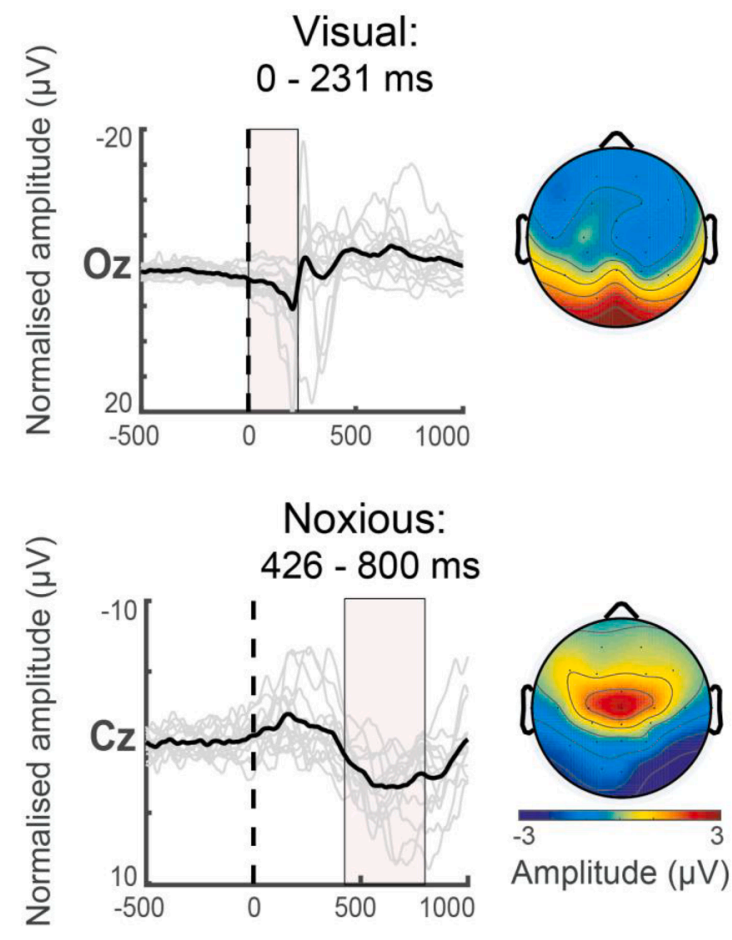

\section{Tactile:} 76 - $218 \mathrm{~ms}$
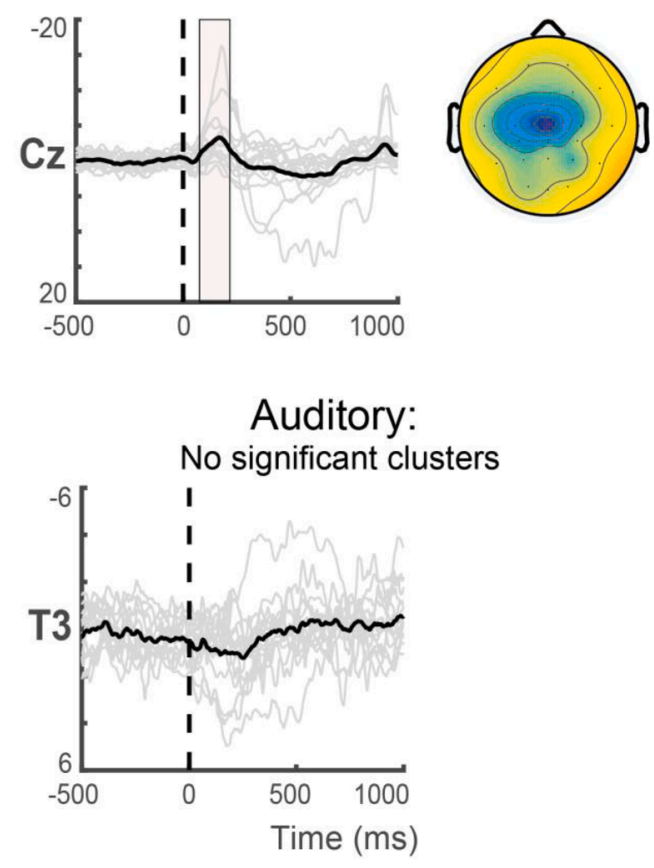

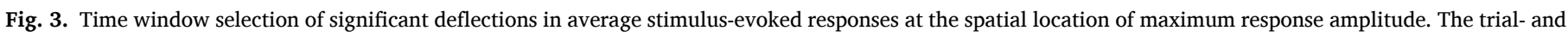

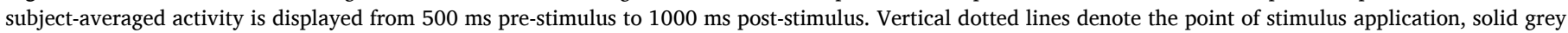

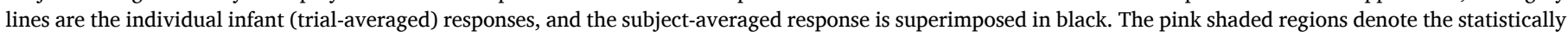

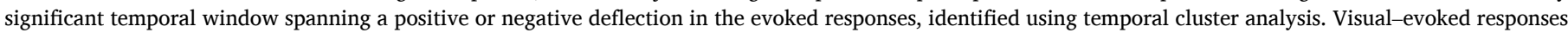

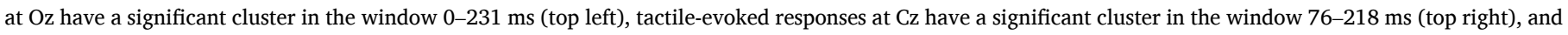

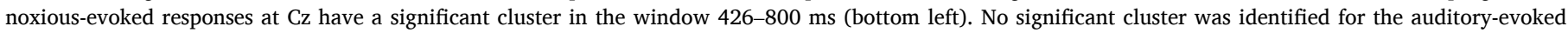

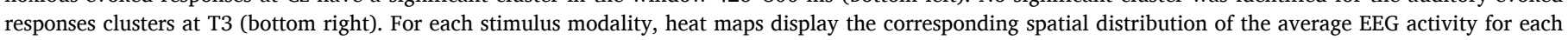

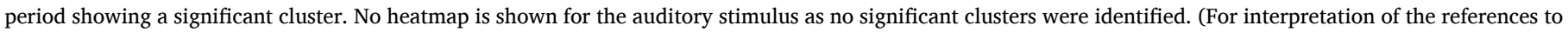
colour in this figure legend, the reader is referred to the web version of this article.) 
VISUAL
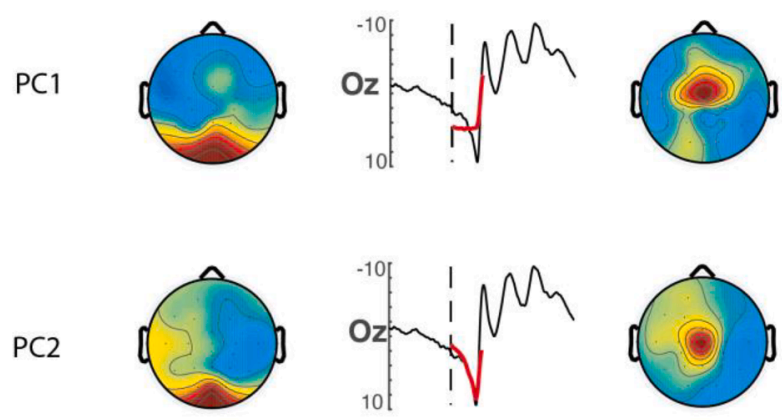

PC2

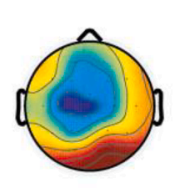

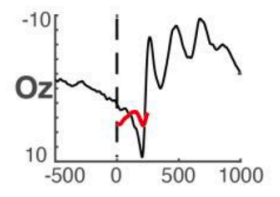

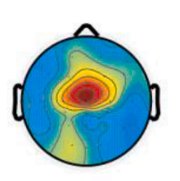

TACTILE<smiles>CCCCN(I)I(I)C(C)(C)C</smiles>
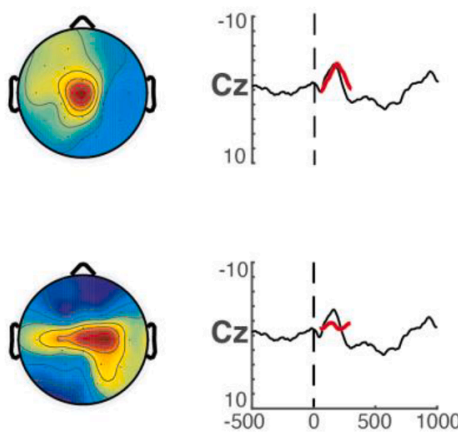
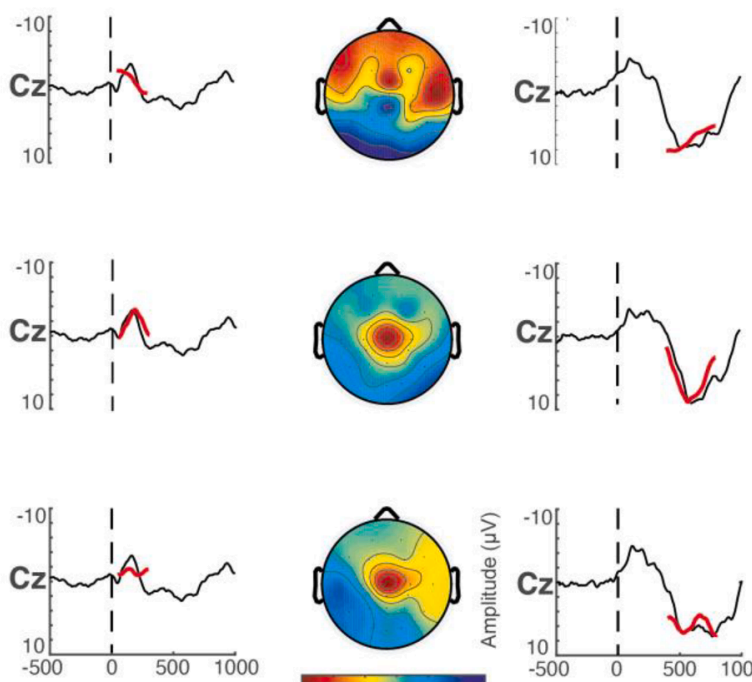

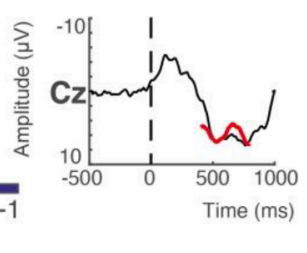

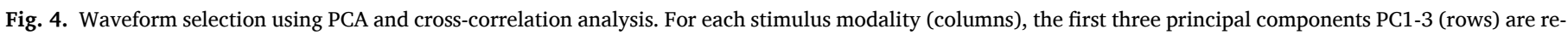

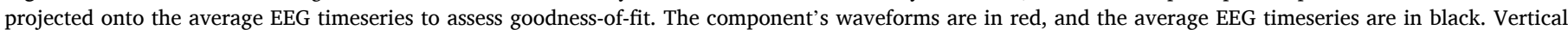

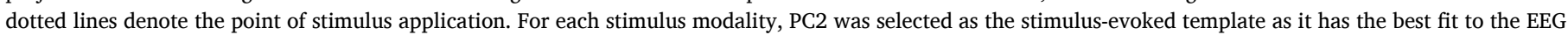

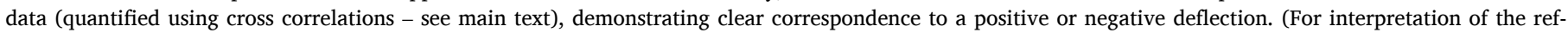
erences to colour in this figure legend, the reader is referred to the web version of this article.)

\subsubsection{Summary of standardised template analysis pipeline for $E E G$ stimulus-evoked feature extraction}

The three stages for feature extraction (Sections 3.1.1 to 3.1.3) were applied consistently across stimulus modalities and identified biophysically sensible template waveforms for visual-, tactile-, and noxiousevoked EEG activity (Fig. 4 row 2). These template vectors can be projected onto novel independent data and scaled for best fit using the spatial and temporal constraints identified in this analysis pipeline outline: visual template applied to electrode $\mathrm{Oz}$ within the $0-250 \mathrm{~ms}$ post-stimulation temporal window; tactile template applied to electrode $\mathrm{Cz}$ within the 50-300 ms post-stimulation temporal window; and noxious template applied to electrode $\mathrm{Cz}$ within the $400-800 \mathrm{~ms}$ poststimulation temporal window.

This scaling factor (vector magnitude) captures the response magnitude of the positive or negative deflection within the defined time windows (with a Woody filtering jitter). While these templates do not capture the entire evoked response waveform, this analysis approach assesses a prominent aspect of the response that exhibits high statistical power, a valuable property in newborn developmental EEG studies with limited sample sizes and limited stimulus trials e.g. single-trial clinically required painful procedures. In addition to magnitude, the template analysis approach can be used to extract non-magnitude features for the relevant deflection such as template latency and goodness-of-fit effects. When applying the templates to independent datasets in the following sections, we focus on magnitude effects due to existing EEG literature highlighting the biological and clinical value of stimulus-evoked response size across stimulus modalities (Hartley et al., 2016; Nevalainen et al., 2008; Whyte et al., 1987).

\subsection{Age-related developmental changes in stimulus-evoked brain activity are detectable using template magnitudes}

Using the spatial and temporal constraints outlined above, we applied the visual, tactile, and noxious templates to stimulus-evoked EEG data in an independent longitudinally studied cohort of very preterm infants $(n=10$, mean GA at birth $=29$ weeks). Infant recordings were separated into three groups according to PMA: $<32$ weeks $(\mathrm{n}=11$, mean PMA at study $=30.4$ weeks), $32-34$ weeks $(\mathrm{n}=7$, mean PMA at study $=32.7$ weeks), 34-37 weeks ( $n=10$, mean PMA at study $=35.1$ weeks). For each stimulus modality, the evoked response template magnitudes were significantly correlated with PMA with variable correlation polarities (Fig. 5A). The visual-evoked response magnitudes were positively correlated with age $\left(R^{2}=0.234\right.$, $p$-value $\left.=0.015\right)$, as were the noxious-evoked response magnitudes $\left(\mathrm{R}^{2}=0.498\right.$, $\mathrm{p}$-value $=$ 0.009). Conversely, the tactile-evoked response magnitudes were negatively correlated with age $\left(R^{2}=0.168\right.$, $p$-value $\left.=0.031\right)$.

The template fit to the average EEG response is displayed for each stimulus modality at each of the three age categories (Fig. 5B timeseries). In general, the visual and tactile templates fit the EEG data well for all age groups. However, the noxious template fit poorly for the youngest ages (PMA $<32$ weeks). A subset of these infants exhibited the positive deflection that the noxious template is designed to fit, while another subset exhibited delta brush responses. Delta brush activity was defined as a slow delta wave with superimposed alpha or beta rhythms (André et al., 2010). These delta brush responses were only seen in response to noxious stimulation in some of the youngest infants (see triangle markers in Fig. 5A Noxious). Due to the mix of positive deflection and delta brush responses, the noxious template magnitude at PMA $<32$ weeks has a group average of approximately zero, suggesting the noxious-evoked delta brush activity is reducing at this developmental period and the positive deflection response is becoming dominant (see Supplementary Fig. 1 for detailed visualisation of the individual EEG evoked responses overlaid with each stimulus-evoked response template).

Fitting the templates to all electrodes across the scalp, the developmentally changing spatial patterns of the stimulus-evoked deflections were qualitatively assessed across age groups (Fig. 5B heat maps). For visual and noxious evoked template magnitudes, which both positively correlated with PMA, the spatial distribution of evoked activity became increasingly localised around the dominant electrode (Oz for visual, $\mathrm{Cz}$ for noxious) as age and magnitude increased. Conversely, for tactile evoked template magnitudes, which were negatively correlated with 
A.

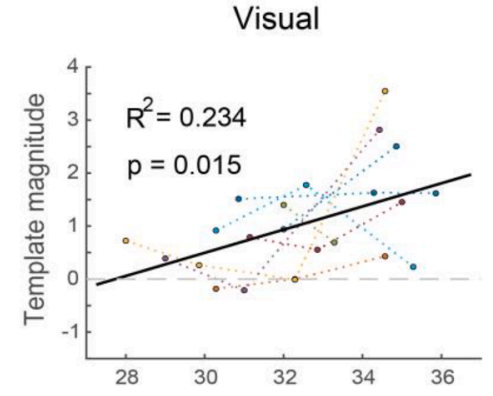

B. PMA (weeks) $<32$

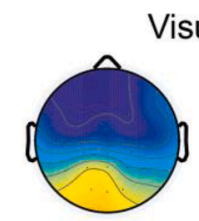

Visual

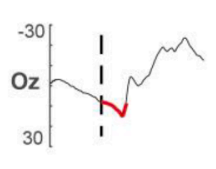

$32-34$

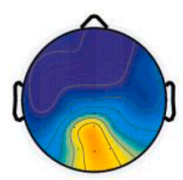

$34-37$
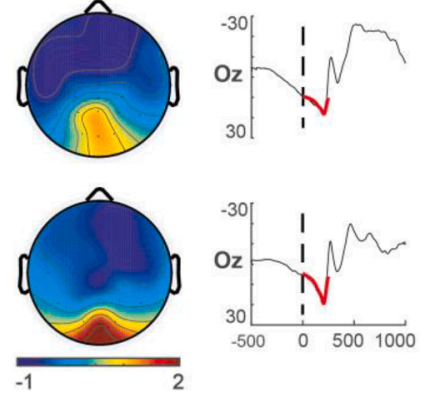

Tactile

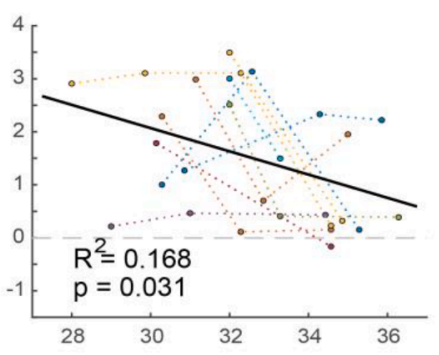

Tactile
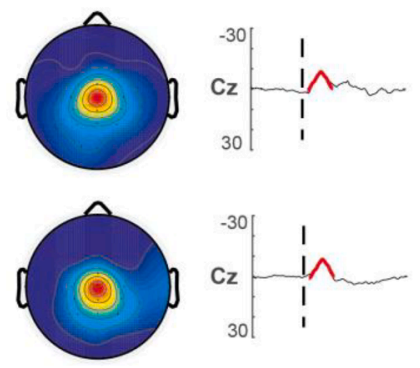

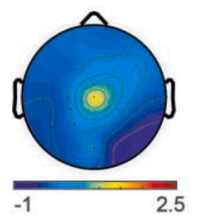

Noxious

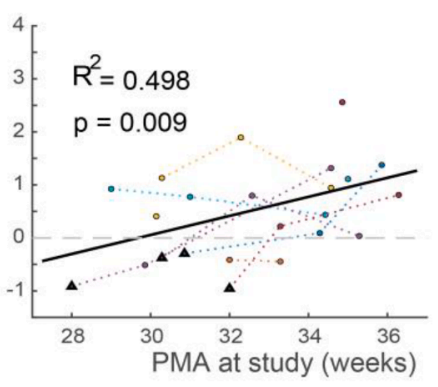

Noxious

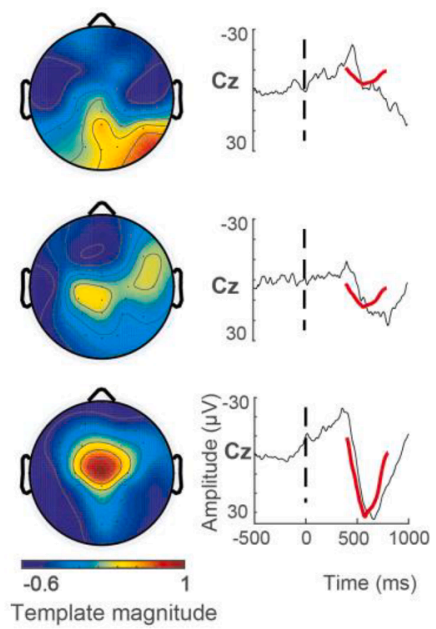

Fig. 5. Longitudinal assessment of age-related developmental changes in stimulus-evoked response template magnitudes. (A) Correlations between template magnitudes and PMA, assessed at the dominant electrode ( $\mathrm{Oz}$ for visual, $\mathrm{Cz}$ for tactile and noxious) using the template analysis approach developed in an independent cohort. Correlations were assessed using linear random effects models. Dotted lines indicate each infant's trajectory plotted in a different colour, with the overall regression line in solid black. The dashed horizontal line denotes the point of zero magnitude. $\mathrm{R}^{2}$ is the regression coefficient of variation (proportion of variance explained), and $\mathrm{p}$ is the associated $\mathrm{p}$-value. The triangle markers below the horizontal zero line of the noxious response plot indicate instances of stimulus-evoked delta brush responses. (B) Heat maps display the developmentally changing spatial patterns of the stimulus-evoked deflections, for qualitative assessment across age groups. The timeseries display the templates (in red) superimposed onto the average EEG response (black) $500 \mathrm{~ms}$ pre-stimulus and $1000 \mathrm{~ms}$ post-stimulus for qualitative assessment of developmentally changing template magnitudes across age groups. Colour bar indicates the template magnitude. (For interpretation of the references to colour in this figure legend, the reader is referred to the web version of this article.)

PMA, the spatial distribution of evoked activity became decreasingly localised around the dominant $\mathrm{Cz}$ electrode as age increased and magnitude decreased.

\subsection{The impact of premature extrauterine development on stimulus- evoked brain activity is detectable using template magnitudes}

To assess the impact of premature extrauterine exposure on sensory development, we compared stimulus-evoked template magnitudes between a very preterm group and a late preterm group. These two groups were matched for PMA (gestational age + postnatal age), but the very preterm group had been developing postnatally for an average of 44 days, while the late preterm group had been developing postnatally for an average of 6 days. Compared to the late preterm infants, the very preterm infants had significantly larger visual-evoked response magnitudes (Cohen's $d=1.287$, p-value $=0.02$ ) and significantly smaller tactile-evoked response magnitudes $(\mathrm{d}=-1.016$, $\mathrm{p}$-value $=0.026)$ (Fig. 6A). There was no significant difference between the groups for the noxious-evoked response magnitudes $(d=0.042$, $p$-value $=0.93)$.

Each stimulus-evoked response template is scaled such that a template magnitude equal to one is equivalent to the expected value that would be recorded from a full term healthy infant (see methods Section 2.2.5). In Fig. 6A, the average absolute value of the template magnitudes for the very preterm and late preterm infants is compared to the benchmark value of one. Relative to the average full term healthy cohort template magnitudes, the very preterm infants exhibited larger visualevoked magnitudes (mean $=1.77$ ) and smaller tactile-evoked magnitudes (mean $=0.80$ ); and the late preterm infants exhibited smaller visual-evoked magnitudes (mean $=0.48$ ) and larger tactile-evoked magnitudes (mean $=2.39$ ).

The template fit to the average EEG response is displayed for each stimulus modality for both infant groups (Fig. 6B timeseries). In general, the visual, tactile, and noxious templates fit the EEG data well for both groups. Fitting the templates to all electrodes across the scalp, differences in spatial patterns of the stimulus-evoked deflections were qualitatively assessed between groups (Fig. 6B heat maps). The topography of response magnitudes did not appear to differ between groups.

\section{Discussion}

This study demonstrates that premature extrauterine exposure has a significant impact on the neural development of visual and tactile systems, detectable in peri-term aged infants and assessed using EEGrecorded brain activity in response to sensory stimulation. Features of the evoked brain activity were identified using a standardised template analysis approach that is a generalisation of a methodology initially developed for the assessment of noxious-evoked EEG brain activity (Hartley et al., 2017). The standardisation of the analysis minimised analytic heterogeneity across the stimulus modalities, and the selection of dominant features of the response signal addressed issues of low 
A.

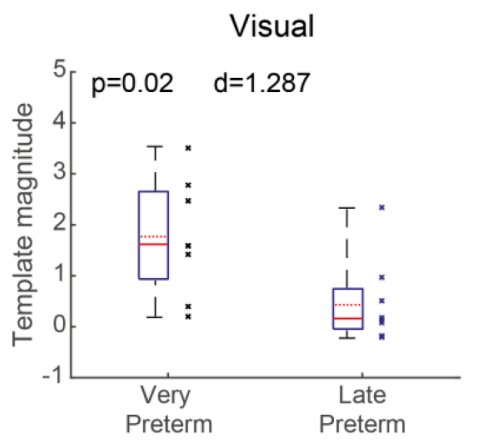

B.

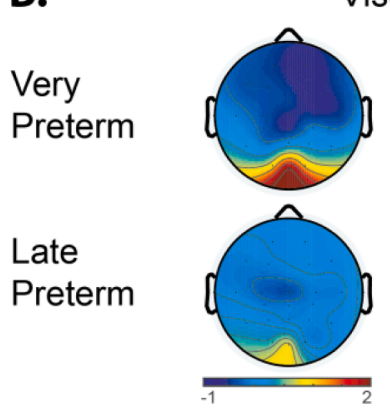

Tactile

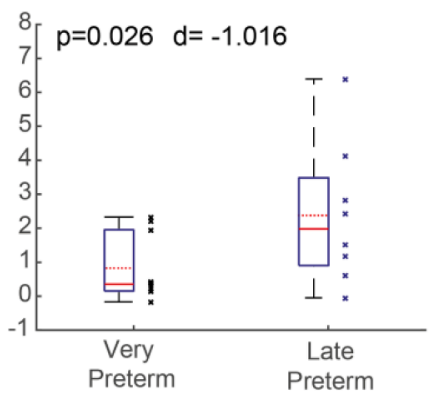

Tactile

\section{Noxious}

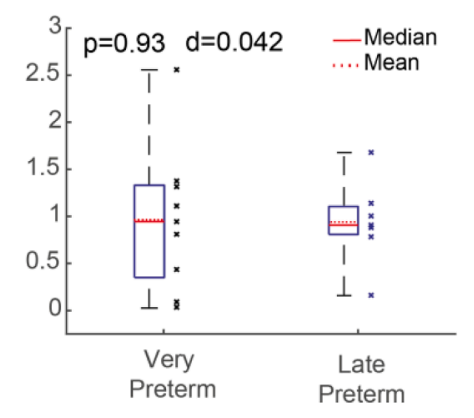

Visual

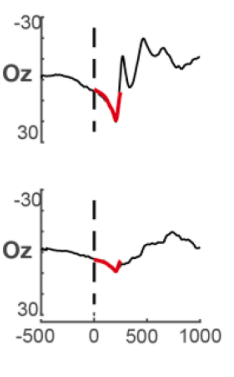

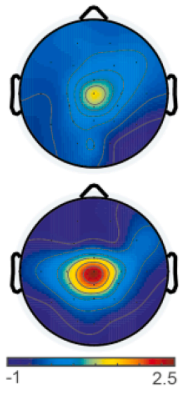

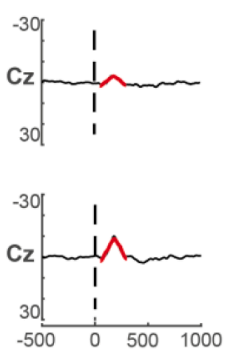

Noxious

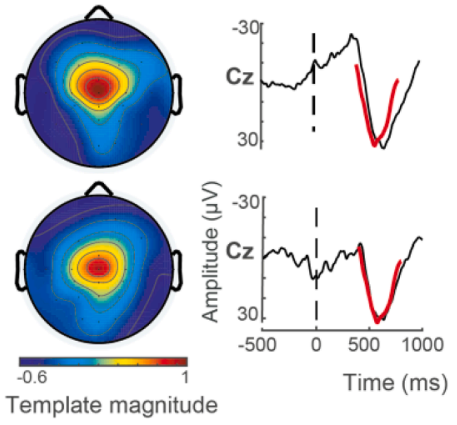

Fig. 6. The impact of premature extrauterine development on stimulus-evoked response template magnitudes. Response magnitudes were assessed using the template analysis approach, and group average response magnitudes were compared between the very preterm infants ( $<32$ weeks) and late preterm infants ( $34-36$ weeks). Both groups were assessed at matched PMA of 34-37 weeks. Visual-evoked template magnitudes were calculated at electrode Oz, and tactile- and noxiousevoked template magnitudes were calculated at electrode Cz. (A) Quantitative comparison of evoked template magnitudes between groups using linear random effects regression model. Compared to the late preterm infants, the very preterm infants had significantly larger visual-evoked response magnitudes (left) and significantly smaller tactile-evoked response magnitudes (middle). There was no significant difference between the groups for the noxious-evoked response magnitudes (right). For all plots, $\mathrm{d}=$ Cohen's d displaying the standardised difference in mean magnitudes; $\mathrm{p}=$ two-sided p-values. (B) Heat maps display the spatial patterns of the stimulus-evoked deflections, for qualitative assessment between groups. The timeseries display the templates (in red) superimposed onto the average EEG response (black) $500 \mathrm{~ms}$ pre-stimulus and $1000 \mathrm{~ms}$ post-stimulus for qualitative assessment of variable response magnitudes between groups. Colour bar indicates the template magnitude scaling. For all stimulus modalities, the templates fit the data well in both groups, and response topography patterns did not appear to be noticeably different between groups. (For interpretation of the references to colour in this figure legend, the reader is referred to the web version of this article.)

statistical power that often accompany infant studies of brain function development due to limited sample sizes and number of stimulus trials e. g. single-trial clinically required painful procedures.

A potential challenge to using the current data-driven approach to feature extraction, which relies on large signal amplitude and survival of statistical thresholding, is the integration of novel template analysis results to previously established features of evoked potentials. The template waveforms identified in the present study for visual-, tactile-, and noxious-evoked activity characterised prominent signal deflections in well-defined temporal windows. Assessing deflection polarity, latency to peak amplitude, and correlation between template magnitude and infant age, the template features bear close correspondence to previously characterised evoked potentials for the three modalities (Slater et al., 2010b; Taylor et al., 1987; Whitehead et al., 2019). The visual template bears close correspondence to the P200 visual-evoked potential, which has an approximate mean peak latency of $211 \mathrm{~ms}$ by term age, and has been shown to increase in amplitude with infant age (Taylor et al., 1987). The tactile template bears close correspondence to the N2 somatosensory-evoked potential, which has an approximate mean peak latency of $160 \mathrm{~ms}$ (Whitehead et al., 2019). While the tactileevoked amplitude of $\mathrm{N} 2$ to foot stimulation was shown to have a nonsignificant association with age, the hand stimulation-evoked N2 amplitude has been shown to decrease with infant age (Whitehead et al., 2019). Finally, the noxious template bears close correspondence to the P560 noxious-evoked potential (Slater et al., 2010b), also known as the P3 potential (Jones et al., 2017), which has an approximate mean peak latency of $560 \mathrm{~ms}$ (Slater et al., 2010b), and has been shown to increase in amplitude with infant age (Hartley et al., 2016). This consistency in polarities, latencies, and age correlations between the current template features and established stimulus-evoked potentials suggests the template analysis approach has selected prominent features of evoked brain activity that can integrate into the existing literature and advance our understanding of the impact of premature exposure to the extrauterine environment on cortical sensory processing. The noxious template derived in the current study also bears a striking correspondence to our previously established noxious template (Hartley et al., 2017), highlighting its reproducibility and the robustness of the template analysis approach applied to the infant population.

Using this standardised template analysis approach, we observed that in longitudinally studied very preterm infants both the visual- and noxious-evoked template magnitudes increased, and tactile-evoked template magnitudes decreased with age, demonstrating the sensitivity of these evoked response features to developmental maturation (Fig. 5). Further, in comparing age-matched infants born in the very preterm and late preterm periods, the very preterm infants had significantly larger visual-evoked and smaller tactile-evoked template magnitudes (Fig. 6), demonstrating the impact of premature extrauterine exposure on both the visual and tactile systems. Given that each stimulus-evoked response template was normalised such that the expected value for full term healthy infants is one, it is interesting to note that the very prematurely born infants had visual responses on average greater than term infants and average tactile responses of lower magnitude than term-born infants. Taken together, these findings suggest that greater premature extrauterine exposure alters the functional 
maturation of neural processes that underpin the visual and tactile systems, as assessed using EEG cortical evoked activity. While alternative interpretations of these results are possible, a plausible and parsimonious interpretation is that exposure to the extrauterine environment may accelerate neural functional maturation. Nevertheless, the factors that underpin the observed differences, such as exposure to adverse sensory stimuli in early life should be investigated.

Accelerated or precocious maturation of neural function due to prematurity has previously been suggested for some sensory modalities as well as spontaneous resting-state activity. A longitudinal study has previously reported that preterm extra-uterine visual experience accelerates maturation of the VEP waveform (Tsuneishi and Casaer, 2000). By studying the decreasing latency of the stimulus-evoked activity with increasing maturation, extrauterine life has also been shown to accelerate maturation of the visual system (Schwindt et al., 2018; Taylor et al., 1987). Prematurity can also precipitate the early onset of binocular vision, assessed using visual evoked EEG activity (Jando et al., 2012). Similarly, premature extrauterine life has been shown to accelerate maturation of the auditory system, assessed using latencies of evoked EEG activity (Cavalcanti et al., 2020). And using auditory verbal stimulation, haemodynamic responses assessed using NIRS had shorter latency as a consequence of early extrauterine exposure, suggesting accelerated maturation of the underlying neural responses, the subsequent haemodynamic response, the neurovascular coupling mechanism, or a combination thereof (Nishida et al., 2008). While these previous findings lend support to an accelerated maturation interpretation of the visual evoked activity observed in the current study, we were unable to assess the influence of prematurity on auditory-evoked cortical activity due to lack of statistically significant evoked responses (Fig. 3). Future optimisation of the nature or delivery of the auditory stimulus will address this issue.

The attenuation of tactile-evoked response magnitudes found in infants born very prematurely in this study is consistent with previous infant studies. Although Maitre and colleagues (Maitre et al., 2017) studied a later potential (P2), they observed that ex-premature infants at term exhibit attenuated tactile-evoked EEG responses compared to termborn infants, which are proportional to the degree of prematurity at birth. A similar but non-significant trend in amplitude was also observed in another infant EEG study (Tombini et al., 2009) and a MEG study similarly reported significant reduction in tactile-evoked responses in ex-premature infants (Nevalainen et al., 2008). In all of these studies, it is unclear whether this represents precocious normal somatosensory development, which mirrors experience-dependent maturation that normally occurs postnatally in term-born infants, or whether these observed effects indicate alternative developmental trajectories. A future longitudinal study of multisensory evoked responses in term-born infants might allow us to address this question. Interestingly, in rats, the process of birth initiates the development of somatosensory map formation and this process is also initiated and accelerated by preterm birth (Toda et al., 2013).

There is little published regarding the influence of premature extrauterine exposure on noxious-evoked cortical activity in the periterm period. To the best of the authors' knowledge, only one EEG study has previously compared age-matched term and ex-premature infants (Slater et al., 2010a), and this study found that infants with greater premature extrauterine exposure exhibited larger noxious-evoked EEG responses. The magnitude of the noxious-evoked cortical activity has previously been shown to be sensitive to developmental maturation (Hartley et al., 2016), and this finding has been replicated here (Fig. 5). These observations suggest the previous findings (Slater et al., 2010a) are consistent with the interpretation that premature extrauterine exposure may result in accelerated maturation of the noxious-evoked cortical response assessed using EEG. However, in the current study, we failed to replicate the observation of a significant difference in noxious-evoked template magnitude between the age-matched very preterm and late preterm infants (Fig. 6).
This lack of a significant difference may be a false negative due to inadequate statistical power. Of the three stimulus modalities used in the cross-sectional study, only the noxious stimulus was a single-trial clinical heel lance, while the visual and tactile stimuli were trains of multi-trial experimental stimuli, likely resulting in the noxious-evoked activity being the noisiest most variable response. Alternatively, the lack of a significant difference may be a true negative - the effect of extra-uterine experience on noxious-evoked responses may be dependent on the degree of exposure to postnatal stressors and insults. In the current study, the ten prematurely-born infants studied in our very preterm cohort were remarkably healthy, with no significant intracranial pathology, no history of surgery, a low burden of pain exposure, and only two infants requiring invasive ventilation for $>24 \mathrm{~h}$. Conversely, the previous study included infants with significant neurological pathology (intraventricular haemorrhages grades 1-4) and greater exposure to invasive ventilation and painful clinical procedures including surgery. Future studies using the standardised template analysis approach directly contrasting two populations of prematurely-born infants matched for both gestational and postnatal ages should explore these various environmental and pathological factors that may impact the maturation of noxious-evoked brain activity.

The influence of premature extrauterine exposure on brain function is not limited to stimulus-evoked activity, but also impacts on restingstate activity. Using amplitude-integrated EEG assessment of ongoing spontaneous brain activity to compare age-matched infants with varying gestational ages, infants born more prematurely displayed advanced maturation compared to less premature infants (Soubasi et al., 2009). Ex-premature infants at term also display some features of accelerated maturation of EEG-sleep organisation, having longer sleep cycles, less fragmentation, and more quiet sleep than their term-born counterparts (Scher, 1997), which may be an adaptive response to the environment but representative of dysmaturity. Resting-state fMRI has also been used to assess the impact of premature extrauterine life and differences in functional connectivity are observable between extrauterine premature infants and in-utero foetuses (De Asis-Cruz et al., 2020). Specifically, the extrauterine infants had greater functional connectivity between brain regions within the auditory (temporal regions), visual (occipital regions), and stress/pain (hippocampal and insular regions) systems, suggesting accelerated maturation of these systems due to extrauterine exposure.

There are several conceptual models in the literature proposed to explain accelerated or precocious maturation of neural function due to premature extrauterine exposure. The stress acceleration hypothesis (Callaghan and Tottenham, 2016), predictive adaptive and other adaptive response hypotheses (Gluckman et al., 2005; Sih, 2011) propose that early life stressors such as premature exposure to the extrauterine environment could result in accelerated maturation along normal developmental trajectories or the adoption of alternative developmental trajectories to adapt to the challenging extrauterine context. They further suggest that the short-term benefits of developmental acceleration and adaptation may not hold long-term and are likely to incur numerous costs. The long-term neurodevelopmental deficits resulting from very preterm birth are well documented and range from motor deficits and cerebral palsy to cognitive impairments and social and emotional difficulties (Anderson and Doyle, 2004; Bhutta et al., 2002; Holsti et al., 2002; Laptook et al., 2005; Marlow et al., 2005; Nosarti et al., 2012; Saigal and Doyle, 2008; Taylor et al., 2004). The short-term costs in neural function detectable using fMRI at termequivalent age for infants born prematurely have also been identified as widespread developmental delays and impairments in functional connectivity and global network measures of integration, segregation, and modularity (Bouyssi-Kobar et al., 2019; Eyre et al., 2021; Scheinost et al., 2016).

The complex variability in reported influences of premature exposure on infant brain function are likely due to heterogeneity across functional system development, brain activity measurement modality, 
and analysis methodology. Maturation of the infant brain's neural systems is both spatially and functionally heterogeneous in timing and rate of development (Cao et al., 2017; Dubois et al., 2014, 2008; Ouyang et al., 2019) resulting in variability in system critical periods and consequent vulnerabilities (Reh et al., 2020). Additionally, unlike EEG and MEG, haemodynamic-based brain assessment modalities such as fMRI and NIRS will not only reflect the influence of prematurity on neural activity but also the impact on cerebral vascular and neurovascular coupling development (Kozberg and Hillman, 2016). Methodological heterogeneity across studies further compounds the challenges in pooling these observations into a unified framework of understanding - see (Botvinik-Nezer et al., 2020) for an informative example of the substantial effects that analytical flexibility can have on scientific conclusions. The approach taken in the current study aimed to address some of these limitations by studying multiple sensory modalities within the same infants, assessing evoked response features both longitudinally and cross-sectionally, while using a standardised analysis methodology across sub-studies. However, interpretational limitations still remain, which future multi-imaging modality studies may address. While simultaneous EEG and fMRI recordings in infants is incredibly challenging, it is possible (Arichi et al., 2017) and would be highly valuable to better understand the associations between prematurity-related effects on infant neurodynamics assessed using EEG and haemodynamics assessed using fMRI, and follow-up childhood sensorimotor and cognitive outcomes to assess the long-lasting effects of these peri-term age brain function differences, if any.

The identification of brain activity features sensitive to the impact of premature extrauterine exposure on the visual and tactile systems highlights the importance of brain-based measures in assessing healthy and disrupted neurodevelopment. The use of EEG features as outcome measures for assessing the efficacy of developmental care interventions is increasing in uptake (Burke, 2018; Neel et al., 2019). Findings consistently indicate positive differences in EEG activity in favour of the intervention group (Als et al., 2011; Kaffashi et al., 2013; McAnulty et al., 2013, 2009; Myers et al., 2015; Welch et al., 2014), and this consistency in findings suggests that neurodevelopmental differences may be detectable neurophysiologically before they manifest behaviourally (Burke, 2018). Previous studies have primarily focused on EEG features of spontaneous brain activity, such as coherence (Als et al., 2011; McAnulty et al., 2013, 2009; Myers et al., 2015), entropy (Kaffashi et al., 2013), and power (Welch et al., 2014). The current study outlines a standardised analysis methodology and outcome measures that can be used for multi-modal EEG evoked activity analysis for a more direct and targeted assessment of multiple sensory system function within an individual infant. Given the sensitivity of the premature infant's neurodevelopment to sensory input (Colonnese et al., 2010; Milh et al., 2007; Wess et al., 2017), and the negative impact prematurity can have across sensory systems of the brain (Wallois et al., 2020), development and application of multi-modal EEG sensory assessment, such as the template analysis of evoked activity, is likely to be a valuable approach to complement resting-state EEG and behavioural assessments when studying the influence of prematurity on neurodevelopment and the efficacy of developmental care interventions.

\section{Conclusions}

We outline a standardised template analysis approach to measure the EEG dominant waveform magnitude evoked by visual, tactile, and noxious stimulation. We observe that the evoked template magnitudes for these three sensory modalities are sensitive to the infant's age at the time of assessment, and that significant differences in neural processing of both visual and tactile stimulation exist between infants born very preterm and late preterm, demonstrating the impact of premature exposure to the extrauterine environment on brain function. Taken together, our findings suggest that premature extrauterine exposure may accelerate maturation of visual and tactile neural systems, and highlight the importance of brain-based EEG measures in assessing healthy and disrupted neurodevelopment. Additionally, we suggest that multi-modal evoked activity analysis, such as the template analysis approach, could complement resting-state EEG and behavioural assessments of the efficacy of developmental care interventions by providing direct and targeted assessment of multiple sensory systems within an individual infant.

\section{Data and code availability statement}

The data that support the study findings are available from the corresponding author upon reasonable request. Due to ethical restrictions, it is appropriate to monitor access and usage of the data as it includes highly sensitive information. Data sharing requests should be directed to rebeccah.slater@paediatrics.ox.ac.uk. The code underpinning template construction is available on GitLab: https://gitlab.com/paediatric_ne uroimaging/stim-template-development. Template fitting to novel data and linear modelling, as detailed in methods, was performed using standard MATLAB commands (https://mathworks.com/products/matla b.html).

\section{CRediT authorship contribution statement}

Gabriela Schmidt Mellado: Conceptualization, Methodology, Investigation, Data curation, Writing - original draft, Visualization. Kirubin Pillay: Methodology, Software, Formal analysis, Data curation, Writing - original draft, Visualization. Eleri Adams: Writing - review \& editing, Supervision. Ana Alarcon: Conceptualization, Writing - review \& editing, Supervision. Foteini Andritsou: Investigation, Writing - review \& editing. Maria M. Cobo: Investigation, Writing - review \& editing. Ria Evans Fry: Investigation, Writing - review \& editing. Sean Fitzgibbon: Writing - review \& editing. Fiona Moultrie: Writing review \& editing. Luke Baxter: Project administration, Validation, Writing - original draft. Rebeccah Slater: Conceptualization, Writing review \& editing, Supervision, Project administration, Funding acquisition.

\section{Declaration of Competing Interest}

The authors declare that they have no known competing financial interests or personal relationships that could have appeared to influence the work reported in this paper.

\section{Acknowledgements}

We thank the neonates and their parents for generously taking part in this study. We thank Marianne van der Vaart, Miranda Buckle, Deniz Gursul, and Shellie Robinson for their assistance during data collection. This study was funded by a Senior Wellcome Research Fellowship awarded to R.S. (207457/Z/17/Z).

\section{Appendix A. Supplementary data}

Supplementary data to this article can be found online at https://doi. org/10.1016/j.nicl.2021.102914.

\section{References}

Adams, J.N., Feldman, H.M., Huffman, L.C., Loe, I.M., 2015. Sensory processing in preterm preschoolers and its association with executive function. Early Hum. Dev. 91 (3), 227-233. https://doi.org/10.1016/j.earlhumdev.2015.01.013.

Als, H., Duffy, F.H., McAnulty, G.B., Fischer, C.B., Kosta, S., Butler, S.C., Parad, R.B., Blickman, J.G., Zurakowski, D., Ringer, S.A., 2011. Is the Newborn Individualized Developmental Care and Assessment Program (NIDCAP) effective for preterm infants with intrauterine growth restriction? J. Perinatol. 31 (2), 130-136. https://doi.org/ 10.1038/jp.2010.81. 
Anderson, P.J., Doyle, L.W., 2004. Executive Functioning in School-Aged Children Who Were Born Very Preterm or With Extremely Low Birth Weight in the 1990s. Pediatrics 114, 50-57. https://doi.org/10.1542/peds.114.1.50.

André, M., Lamblin, M.-D., d'Allest, A.M., Curzi-Dascalova, L., Moussalli-Salefranque, F., Nguyen The Tich, S., Vecchierini-Blineau, M.-F., Wallois, F., Walls-Esquivel, E., Plouin, P., 2010. Electroencephalography in premature and full-term infants. Developmental features and glossary. Neurophysiologie Clinique/Clin. Neurophysiol. 40 (2), 59-124. https://doi.org/10.1016/j.neucli.2010.02.002.

Arichi, T., Whitehead, K., Barone, G., Pressler, R., Padormo, F., Edwards, A.D., Fabrizi, L., 2017. Localization of spontaneous bursting neuronal activity in the preterm human brain with simultaneous EEG-fMRI. Elife 6. https://doi.org/10.7554/ eLife.27814.

Baxter, L., Moultrie, F., Fitzgibbon, S., Aspbury, M., Mansfield, R., Bastiani, M., Rogers, R., Jbabdi, S., Duff, E., Slater, R., 2021. Functional and diffusion MRI reveal the neurophysiological basis of neonates' noxious-stimulus evoked brain activity. Nat. Commun. 12, 2744. https://doi.org/10.1038/s41467-021-22960-0.

Bhutta, A.T., Cleves, M.A., Casey, P.H., Cradock, M.M., Anand, K.J.S., 2002. Cognitive and behavioral outcomes of school-aged children who were born preterm: a metaanalysis. JAMA 288, 728-737. https://doi.org/10.1001/jama.288.6.728.

Blencowe, H., Lee, A.C.C., Cousens, S., Bahalim, A., Narwal, R., Zhong, N., Chou, D., Say, L., Modi, N., Katz, J., Vos, T., Marlow, N., Lawn, J.E., 2013. Preterm birthassociated neurodevelopmental impairment estimates at regional and global levels for 2010. Pediatr. Res. 74 (Suppl 1), 17-34. https://doi.org/10.1038/pr.2013.204.

Botvinik-Nezer, R., Holzmeister, F., Camerer, C.F., Dreber, A., Huber, J., Johannesson, M., Kirchler, M., Iwanir, R., Mumford, J.A., Adcock, R.A., Avesani, P., Baczkowski, B.M., Bajracharya, A., Bakst, L., Ball, S., Barilari, M., Bault, N., Beaton, D., Beitner, J., Benoit, R.G., Berkers, R.M.W.J., Bhanji, J.P., Biswal, B.B. Bobadilla-Suarez, S., Bortolini, T., Bottenhorn, K.L., Bowring, A., Braem, S., Brooks, H.R., Brudner, E.G., Calderon, C.B., Camilleri, J.A., Castrellon, J.J., Cecchetti, L., Cieslik, E.C., Cole, Z.J., Collignon, O., Cox, R.W., Cunningham, W.A., Czoschke, S., Dadi, K., Davis, C.P., Luca, A.D., Delgado, M.R., Demetriou, L., Dennison, J.B., Di, X., Dickie, E.W., Dobryakova, E., Donnat, C.L., Dukart, J., Duncan, N.W., Durnez, J., Eed, A., Eickhoff, S.B., Erhart, A., Fontanesi, L., Fricke, G. M., Fu, S., Galván, A., Gau, R., Genon, S., Glatard, T., Glerean, E., Goeman, J.J., Golowin, S.A.E., González-García, C., Gorgolewski, K.J., Grady, C.L., Green, M.A., Guassi Moreira, J.F., Guest, O., Hakimi, S., Hamilton, J.P., Hancock, R., Handjaras, G., Harry, B.B., Hawco, C., Herholz, P., Herman, G., Heunis, S., Hoffstaedter, F., Hogeveen, J., Holmes, S., Hu, C.-P., Huettel, S.A., Hughes, M.E., Iacovella, V., Iordan, A.D., Isager, P.M., Isik, A.I., Jahn, A., Johnson, M.R., Johnstone, T., Joseph, M.J.E., Juliano, A.C., Kable, J.W., Kassinopoulos, M., Koba, C., Kong, X.-Z., Koscik, T.R., Kucukboyaci, N.E., Kuhl, B.A., Kupek, S., Laird, A. R., Lamm, C., Langner, R., Lauharatanahirun, N., Lee, H., Lee, S., Leemans, A., Leo, A., Lesage, E., Li, F., Li, M.Y.C., Lim, P.C., Lintz, E.N., Liphardt, S.W., Losecaat Vermeer, A.B., Love, B.C., Mack, M.L., Malpica, N., Marins, T., Maumet, C., McDonald, K., McGuire, J.T., Melero, H., Méndez Leal, A.S., Meyer, B., Meyer, K.N., Mihai, G., Mitsis, G.D., Moll, J., Nielson, D.M., Nilsonne, G., Notter, M.P., Olivetti, E., Onicas, A.I., Papale, P., Patil, K.R., Peelle, J.E., Pérez, A., Pischedda, D., Poline, J.-B., Prystauka, Y., Ray, S., Reuter-Lorenz, P.A., Reynolds, R.C., Ricciardi, E., Rieck, J.R. Rodriguez-Thompson, A.M., Romyn, A., Salo, T., Samanez-Larkin, G.R., SanzMorales, E., Schlichting, M.L., Schultz, D.H., Shen, Q., Sheridan, M.A., Silvers, J.A., Skagerlund, K., Smith, A., Smith, D.V., Sokol-Hessner, P., Steinkamp, S.R., Tashjian, S.M., Thirion, B., Thorp, J.N., Tinghög, G., Tisdall, L., Tompson, S.H., ToroSerey, C., Torre Tresols, J.J., Tozzi, L., Truong, V., Turella, L., van 't Veer, A.E., Verguts, T., Vettel, J.M., Vijayarajah, S., Vo, K., Wall, M.B., Weeda, W.D., Weis, S., White, D.J., Wisniewski, D., Xifra-Porxas, A., Yearling, E.A., Yoon, S., Yuan, R., Yuen, K.S.L., Zhang, L., Zhang, X., Zosky, J.E., Nichols, T.E., Poldrack, R.A., Schonberg, T., 2020. Variability in the analysis of a single neuroimaging dataset by many teams. Nature 582, 84-88. https://doi.org/10.1038/s41586-020-2314-9.

Bouyssi-Kobar, M., Asis-Cruz, J.D., Murnick, J., Chang, T., Limperopoulos, C., 2019. Altered Functional Brain Network Integration, Segregation, and Modularity in Infants Born Very Preterm at Term-Equivalent Age. J. Pediatr. 213, 13-21.e1. https://doi.org/10.1016/j.jpeds.2019.06.030.

Burke, Sara, 2018. Systematic review of developmental care interventions in the neonatal intensive care unit since 2006. J. Child Health Care 22 (2), 269-286. https://doi.org/10.1177/1367493517753085.

Callaghan, B.L., Tottenham, N., 2016. The Stress Acceleration Hypothesis: Effects of early-life adversity on emotion circuits and behavior. Curr. Opin. Behav. Sci. 7, 76-81. https://doi.org/10.1016/j.cobeha.2015.11.018.

Cao, M., He, Y., Dai, Z., Liao, X., Jeon, T., Ouyang, M., Chalak, L., Bi, Y., Rollins, N. Dong, Q., Huang, H., 2017. Early Development of Functional Network Segregation Revealed by Connectomic Analysis of the Preterm Human Brain. Cereb. Cortex 27, 1949-1963. https://doi.org/10.1093/cercor/bhw038.

Cavalcanti, H.G., da Silva Nunes, A.D., da Cunha, B.K.S., de Freitas Alvarenga, K., Balen, S.A., Pereira, A., 2020. Early exposure to environment sounds and the development of cortical auditory evoked potentials of preterm infants during the first 3 months of life. BMC Res. Notes 13, 303. https://doi.org/10.1186/s13104-020 05129-8.

Cobo, M.M., Hartley, C., Gursul, D., Andritsou, F., van der Vaart, M., Schmidt Mellado, G., Baxter, L., Duff, E.P., Buckle, M., Evans Fry, R., Green, G., Hoskin, A. Rogers, R., Adams, E., Moultrie, F., Slater, R., 2021. Quantifying noxious-evoked baseline sensitivity in neonates to optimise analgesic trials. eLife 10, e65266. https://doi.org/10.7554/eLife.65266.

Colonnese, Matthew, Khazipov, Rustem, 2012. Spontaneous activity in developing sensory circuits: Implications for resting state fMRI. NeuroImage, Connectivity 62 (4), 2212-2221. https://doi.org/10.1016/j.neuroimage.2012.02.046.
Colonnese, Matthew T., Kaminska, Anna, Minlebaev, Marat, Milh, Mathieu, Bloem, Bernard, Lescure, Sandra, Moriette, Guy, Chiron, Catherine, BenAri, Yehezkel, Khazipov, Rustem, 2010. A Conserved Switch in Sensory Processing Prepares Developing Neocortex for Vision. Neuron 67 (3), 480-498. https://doi.org/ 10.1016/j.neuron.2010.07.015.

Courtois, E., Droutman, S., Magny, J.-F., Merchaoui, Z., Durrmeyer, X., Roussel, C. Biran, V., Eleni, S., Vottier, G., Renolleau, S., Desfrere, L., Castela, F., Boimond, N., Mellah, D., Bolot, P., Coursol, A., Brault, D., Chappuy, H., Cimerman, P., Anand, K.J. S., Carbajal, R., 2016. Epidemiology and neonatal pain management of heelsticks in intensive care units: EPIPPAIN 2, a prospective observational study. Int. J. Nurs. Stud. 59, 79-88. https://doi.org/10.1016/j.ijnurstu.2016.03.014.

De Asis-Cruz, Josepheen, Kapse, Kushal, Basu, Sudeepta K., Said, Mariam, Scheinost, Dustin, Murnick, Jonathan, Chang, Taeun, du Plessis, Adre, Limperopoulos, Catherine, 2020. Functional brain connectivity in ex utero premature infants compared to in utero fetuses. NeuroImage 219, 117043. https:// doi.org/10.1016/j.neuroimage.2020.117043.

Dubois, J., Dehaene-Lambertz, G., 2015. In: Brain Mapping. Elsevier, pp. 11-19. https:// doi.org/10.1016/B978-0-12-397025-1.00194-9.

Dubois, J., Dehaene-Lambertz, G., Kulikova, S., Poupon, C., Hüppi, P.S., HertzPannier, L., 2014. The early development of brain white matter: a review of imaging studies in fetuses, newborns and infants. Neuroscience 276, 48-71. https://doi.org/ 10.1016/j.neuroscience.2013.12.044.

Dubois, Jessica, Dehaene-Lambertz, Ghislaine, Perrin, Muriel, Mangin, Jean-François, Cointepas, Yann, Duchesnay, Edouard, Le Bihan, Denis, Hertz-Pannier, Lucie, 2008. Asynchrony of the early maturation of white matter bundles in healthy infants: quantitative landmarks revealed noninvasively by diffusion tensor imaging. Hum. Brain Mapp. 29 (1), 14-27. https://doi.org/10.1002/(ISSN)1097-019310.1002/ hbm.v29:110.1002/hbm.20363.

Eyre, M., Fitzgibbon, S.P., Ciarrusta, J., Cordero-Grande, L., Price, A.N., Poppe, T., Schuh, A., Hughes, E., O'Keeffe, C., Brandon, J., Cromb, D., Vecchiato, K., Andersson, J., Duff, E.P., Counsell, S.J., Smith, S.M., Rueckert, D., Hajnal, J.V., Arichi, T., O'Muircheartaigh, J., Batallea, D., Edwards, A.D., 2021. The Developing Human Connectome Project: typical and disrupted perinatal functional connectivity. Brain. https://doi.org/10.1093/brain/awab118.

Gluckman, P.D., Cutfield, W., Hofman, P., Hanson, M.A., 2005. The fetal, neonatal, and infant environments - the long-term consequences for disease risk. Early Human Development, Selected contributions from the Neonatal Update 2004 (81), 51-59. https://doi.org/10.1016/j.earlhumdev.2004.10.003.

Green, Gabrielle, Hartley, Caroline, Hoskin, Amy, Duff, Eugene, Shriver, Adam, Wilkinson, Dominic, Adams, Eleri, Rogers, Richard, Moultrie, Fiona, Slater, Rebeccah, 2019. Behavioural discrimination of noxious stimuli in infants is dependent on brain maturation. Pain 160 (2), 493-500. https://doi.org/10.1097/j pain.0000000000001425.

Gursul, Deniz, Goksan, Sezgi, Hartley, Caroline, Mellado, Gabriela Schmidt, Moultrie, Fiona, Hoskin, Amy, Adams, Eleri, Hathway, Gareth, Walker, Susannah, McGlone, Francis, Slater, Rebeccah, 2018. Stroking modulates noxious-evoked brain activity in human infants. Curr. Biol. 28 (24), R1380-R1381. https://doi.org/ 10.1016/j.cub.2018.11.014.

Hartley, Caroline, Baxter, Luke, Moultrie, Fiona, Purdy, Ryan, Bhatt, Aomesh, Rogers, Richard, Patel, Chetan, Adams, Eleri, Slater, Rebeccah, 2021. Predicting severity of adverse cardiorespiratory effects of morphine in premature infants: a post hoc analysis of Procedural Pain in Premature Infants trial data. Br. J. Anaesth. 126 (4), e133-e135. https://doi.org/10.1016/j.bja.2020.10.034.

Hartley, Caroline, Duff, Eugene P., Green, Gabrielle, Mellado, Gabriela Schmidt, Worley, Alan, Rogers, Richard, Slater, Rebeccah, 2017. Nociceptive brain activity as a measure of analgesic efficacy in infants. Sci. Transl. Med. 9 (388) https://doi.org/ 10.1126/scitranslmed.aah6122.

Hartley, C., Goksan, S., Poorun, R., Brotherhood, K., Mellado, G.S., Moultrie, F., Rogers, R., Adams, E., Slater, R., 2015. The relationship between nociceptive brain activity, spinal reflex withdrawal and behaviour in newborn infants. Sci. Rep. 5, 12519. https://doi.org/10.1038/srep12519.

Hartley, Caroline, Moultrie, Fiona, Gursul, Deniz, Hoskin, Amy, Adams, Eleri, Rogers, Richard, Slater, Rebeccah, 2016. Changing Balance of Spinal Cord Excitability and Nociceptive Brain Activity in Early Human Development. Curr. Biol. 26 (15), 1998-2002. https://doi.org/10.1016/j.cub.2016.05.054.

Hartley, Caroline, Moultrie, Fiona, Hoskin, Amy, Green, Gabrielle, Monk, Vaneesha, Bell, Jennifer L, King, Andrew R, Buckle, Miranda, van der Vaart, Marianne, Gursul, Deniz, Goksan, Sezgi, Juszczak, Edmund, Norman, Jane E, Rogers, Richard, Patel, Chetan, Adams, Eleri, Slater, Rebeccah, 2018. Analgesic efficacy and safety of morphine in the Procedural Pain in Premature Infants (Poppi) study: randomised placebo-controlled trial. Lancet 392 (10164), 2595-2605. https://doi.org/10.1016/ S0140-6736(18)31813-0.

Holsti, Liisa, Grunau, Ruth V.E., Whitfield, Michael F., 2002. Developmental coordination disorder in extremely low birth weight children at nine years. J. Dev. Behav. Pediatr. 23 (1), 9-15. https://doi.org/10.1097/00004703-20020200000002.

Iannetti, G.D., Baumgärtner, U., Tracey, I., Treede, R.D., Magerl, W., 2013. Pinprickevoked brain potentials: a novel tool to assess central sensitization of nociceptive pathways in humans. J. Neurophysiol. 110 (5), 1107-1116. https://doi.org/ 10.1152/jn.00774.2012.

Jando, G., Miko-Barath, E., Marko, K., Hollody, K., Torok, B., Kovacs, I., 2012. Earlyonset binocularity in preterm infants reveals experience-dependent visual development in humans. Proc. Natl. Acad. Sci. U. S. A. 109 (27), 11049-11052. https://doi.org/10.1073/pnas.1203096109.

Jones, Laura, Fabrizi, Lorenzo, Laudiano-Dray, Maria, Whitehead, Kimberley, Meek, Judith, Verriotis, Madeleine, Fitzgerald, Maria, 2017. Nociceptive Cortical 
Activity Is Dissociated from Nociceptive Behavior in Newborn Human Infants under Stress. Curr. Biol. 27 (24), 3846-3851.e3. https://doi.org/10.1016/j. cub.2017.10.063.

Kaffashi, F., Scher, M.S., Ludington-Hoe, S.M., Loparo, K.A., 2013. An analysis of the kangaroo care intervention using neonatal EEG complexity: A preliminary study. Clin. Neurophysiol. 124 (2), 238-246. https://doi.org/10.1016/j. clinph.2012.06.021.

Kasser, S., Hartley, C., Rickenbacher, H., Klarer, N., Depoorter, A., Datta, A.N., Cobo, M. M., Goksan, S., Hoskin, A., Magerl, W., Huhn, E.A., Green, G., Slater, R., Wellmann, S., 2019. Birth experience in newborn infants is associated with changes in nociceptive sensitivity. Sci. Rep. 9, 4117. https://doi.org/10.1038/s41598-01940650-2.

Kozberg, M., Hillman, E., 2016. Neurovascular coupling and energy metabolism in the developing brain. Prog. Brain Res. 225, 213-242. https://doi.org/10.1016/bs. pbr.2016.02.002.

Laptook, A.R., O’Shea, T.M., Shankaran, S., Bhaskar, B., Neonatal Network, N.I.C.H.D., 2005. Adverse neurodevelopmental outcomes among extremely low birth weight infants with a normal head ultrasound: prevalence and antecedents. Pediatrics 115, 673-680. https://doi.org/10.1542/peds.2004-0667.

Leikos, Susanna, Tokariev, Anton, Koolen, Ninah, Nevalainen, Päivi, Vanhatalo, Sampsa, 2020. Cortical responses to tactile stimuli in preterm infants. Eur. J. Neurosci. 51 (4), 1059-1073. https://doi.org/10.1111/ejn.v51.410.1111/ejn.14613.

Magerl, W., Fuchs, P.N., Meyer, R.A., Treede, R.D., 2001. Roles of capsaicin-insensitive nociceptors in cutaneous pain and secondary hyperalgesia. Brain 124, 1754-1764. https://doi.org/10.1093/brain/124.9.1754.

Maitre, Nathalie L., Key, Alexandra P., Chorna, Olena D., Slaughter, James C. Matusz, Pawel J., Wallace, Mark T., Murray, Micah M., 2017. The Dual Nature of Early-Life Experience on Somatosensory Processing in the Human Infant Brain. Curr. Biol. 27 (7), 1048-1054. https://doi.org/10.1016/j.cub.2017.02.036.

Majnemer, Annette, Rosenblatt, Bernard, 1996. Evoked potentials as predictors of outcome in neonatal intensive care unit survivors: review of the literature. Pediatr. Neurol. 14 (3), 189-195. https://doi.org/10.1016/0887-8994(96)00049-5.

Maris, Eric, Oostenveld, Robert, 2007. Nonparametric statistical testing of EEG- and MEG-data. J. Neurosci. Methods 164 (1), 177-190. https://doi.org/10.1016/j. jneumeth.2007.03.024.

Marlow, Neil, Wolke, Dieter, Bracewell, Melanie A., Samara, Muthanna, 2005. Neurologic and developmental disability at six years of age after extremely preterm birth. N. Engl. J. Med. 352 (1), 9-19. https://doi.org/10.1056/NEJMoa041367.

McAnulty, G., Duffy, F.H., Butler, S., Parad, R., Ringer, S., Zurakowski, D., Als, H., 2009. Individualized developmental care for a large sample of very preterm infants: health, neurobehaviour and neurophysiology. Acta Paediatr. 98, 1920-1926. https://doi. org/10.1111/j.1651-2227.2009.01492.x.

McAnulty, G., Duffy, F.H., Kosta, S., Weisenfeld, N.I., Warfield, S.K., Butler, S.C., Alidoost, M., Bernstein, J.H., Robertson, R., Zurakowski, D., Als, H., 2013. Schoolage effects of the newborn individualized developmental care and assessment program for preterm infants with intrauterine growth restriction: preliminary findings. BMC Pediatr. 13, 25. https://doi.org/10.1186/1471-2431-13-25.

McMahon, E., Wintermark, P., Lahav, A., 2012. Auditory brain development in premature infants: the importance of early experience. Ann. N. Y. Acad. Sci. 1252, 17-24. https://doi.org/10.1111/j.1749-6632.2012.06445.x.

Milh, M., Kaminska, A., Huon, C., Lapillonne, A., Ben-Ari, Y., Khazipov, R., 2007. Rapid cortical oscillations and early motor activity in premature human neonate. Cereb. Cortex 17, 1582-1594. https://doi.org/10.1093/cercor/bhl069.

Myers, M.M., Grieve, P.G., Stark, R.I., Isler, J.R., Hofer, M.A., Yang, J., Ludwig, R.J. Welch, M.G., 2015. Family Nurture Intervention in preterm infants alters frontal cortical functional connectivity assessed by EEG coherence. Acta Paediatr. 104 (7), 670-677. https://doi.org/10.1111/apa.2015.104.issue-710.1111/apa.13007.

Neel, M.L., Yoder, P., Matusz, P.J., Murray, M.M., Miller, A., Burkhardt, S., Emery, L., Hague, K., Pennington, C., Purnell, J., Lightfoot, M., Maitre, N.L., 2019. Randomized controlled trial protocol to improve multisensory neural processing, language and motor outcomes in preterm infants. BMC Pediatr. 19, 81. https://doi.org/10.1186/ s12887-019-1455-1.

Nevalainen, Päivi, Pihko, Elina, Metsäranta, Marjo, Andersson, Sture, Autti, Taina, Lauronen, Leena, 2008. Does very premature birth affect the functioning of the somatosensory cortex? - A magnetoencephalography study. Int. J. Psychophysiol. MEG Paediatr. 68 (2), 85-93. https://doi.org/10.1016/j.ijpsycho.2007.10.014.

Nishida, Tomoko, Kusaka, Takashi, Isobe, Kenichi, Ijichi, Sonoko, Okubo, Kensuke, Iwase, Takashi, Kawada, Kou, Namba, Masanori, Imai, Tadashi, Itoh, Susumu, 2008 Extrauterine environment affects the cortical responses to verbal stimulation in preterm infants. Neurosci. Lett. 443 (1), 23-26. https://doi.org/10.1016/j. neulet.2008.07.035.

Nosarti, C., Reichenberg, A., Murray, R.M., Cnattingius, S., Lambe, M.P., Yin, L., MacCabe, J., Rifkin, L., Hultman, C.M., 2012. Preterm birth and psychiatric disorders in young adult life. Arch. Gen. Psychiatry 69, E1-8. https://doi.org/ 10.1001/archgenpsychiatry.2011.1374.

Ouyang, M., Dubois, J., Yu, Q., Mukherjee, P., Huang, H., 2019. Delineation of early brain development from fetuses to infants with diffusion MRI and beyond. Neuroimage 185, 836-850. https://doi.org/10.1016/j.neuroimage.2018.04.017.

Pineda, Roberta G., Neil, Jeff, Dierker, Donna, Smyser, Christopher D., Wallendorf, Michael, Kidokoro, Hiroyuki, Reynolds, Lauren C., Walker, Stephanie, Rogers, Cynthia, Mathur, Amit M., Van Essen, David C., Inder, Terrie, 2014. Alterations in Brain Structure and Neurodevelopmental Outcome in Preterm Infants Hospitalized in Different Neonatal Intensive Care Unit Environments. J. Pediatr. 164 (1), 52-60.e2. https://doi.org/10.1016/j.jpeds.2013.08.047.

Reh, Rebecca K., Dias, Brian G., Nelson, Charles A., Kaufer, Daniela, Werker, Janet F., Kolb, Bryan, Levine, Joel D., Hensch, Takao K., 2020. Critical period regulation across multiple timescales. PNAS 117 (38), 23242-23251. https://doi.org/10.1073/ pnas. 1820836117.

Martins Ribeiro, Flávia, Mamede Carvallo, Renata, 2008. Tone-evoked ABR in full-term and preterm neonates with normal hearing. Int. J. Audiol. 47 (1), 21-29. https://doi. org/10.1080/14992020701643800.

Rosenberg, T., Flage, T., Hansen, E., Riise, R., Rudanko, S.L., Viggosson, G., Tornqvist, K., 1996. Incidence of registered visual impairment in the Nordic child population. Br. J. Ophthalmol. 80 (1), 49-53. https://doi.org/10.1136/bjo.80.1.49.

Saigal, Saroj, Doyle, Lex W., 2008. An overview of mortality and sequelae of preterm birth from infancy to adulthood. Lancet 371 (9608), 261-269. https://doi.org/ 10.1016/S0140-6736(08)60136-1.

Scheinost, Dustin, Kwon, Soo Hyun, Shen, Xilin, Lacadie, Cheryl, Schneider, Karen C., Dai, Feng, Ment, Laura R., Constable, R. Todd, 2016. Preterm birth alters neonatal, functional rich club organization. Brain Struct. Funct. 221 (6), 3211-3222. https:// doi.org/10.1007/s00429-015-1096-6.

Scher, Mark S., 1997. Neurophysiological assessment of brain function and maturation. II. A measure of brain dysmaturity in healthy preterm neonates. Pediatr. Neurol. 16 (4), 287-295. https://doi.org/10.1016/S0887-8994(96)00009-4.

Schwindt, Eva, Giordano, Vito, Rona, Zsofia, Czaba-Hnizdo, Christine, Olischar, Monika, Waldhoer, Thomas, Werther, Tobias, Fuiko, Renate, Berger, Angelika, KlebermassSchrehof, Katrin, 2018. The impact of extrauterine life on visual maturation in extremely preterm born infants. Pediatr. Res. 84 (3), 403-410. https://doi.org/ 10.1038/s41390-018-0084-y.

Sih, Andrew, 2011. Effects of early stress on behavioral syndromes: An integrated adaptive perspective. Neurosci. Biobehav. Rev. Resilience Adapt. Aspects Stress Neurobehav. Dev. 35 (7), 1452-1465. https://doi.org/10.1016/j. neubiorev.2011.03.015.

Slater, Rebeccah, Fabrizi, Lorenzo, Worley, Alan, Meek, Judith, Boyd, Stewart, Fitzgerald, Maria, 2010a. Premature infants display increased noxious-evoked neuronal activity in the brain compared to healthy age-matched term-born infants. Neuroimage 52 (2), 583-589. https://doi.org/10.1016/j.neuroimage.2010.04.253.

Slater, R., Worley, A., Fabrizi, L., Roberts, S., Meek, J., Boyd, S., Fitzgerald, M., 2010b. Evoked potentials generated by noxious stimulation in the human infant brain. Eur. J. Pain 14, 321-326. https://doi.org/10.1016/j.ejpain.2009.05.005.

Smith, Gillian C., Gutovich, Jordan, Smyser, Christopher, Pineda, Roberta, Newnham, Carol, Tjoeng, Tiong H., Vavasseur, Claudine, Wallendorf, Michael, Neil, Jeffrey, Inder, Terrie, 2011. Neonatal intensive care unit stress is associated with brain development in preterm infants. Ann. Neurol. 70 (4), 541-549. https:// doi.org/10.1002/ana.v70.410.1002/ana.22545.

Soubasi, Vassiliki, Mitsakis, Konstantinos, Nakas, Christos T., Petridou, Stella, Sarafidis, Kosmas, Griva, Myrsini, Agakidou, Eleni, Drossou, Vassiliki, 2009. The influence of extrauterine life on the aEEG maturation in normal preterm infants. Early Human Dev. 85 (12), 761-765. https://doi.org/10.1016/j. earlhumdev.2009.10.004.

Taylor, H. Gerry, Minich, Nori M., Klein, Nancy, Hack, Maureen, 2004. Longitudinal outcomes of very low birth weight: neuropsychological findings. J. Int. Neuropsychol. Soc. 10 (2), 149-163. https://doi.org/10.1017/ S1355617704102038.

Taylor, M.J., Menzies, R., MacMillan, L.J., Whyte, H.E., 1987. VEPs in normal full-term and premature neonates: longitudinal versus cross-sectional data. Electroencephalogr. Clin. Neurophysiol./Evoked Potentials Section 68 (1), 20-27. https://doi.org/10.1016/0168-5597(87)90066-9.

Taylor, M.J., Murphy, W.J., Whyte, H.E., 1992. Prognostic reliability of somatosensory and visual evoked potentials of asphyxiated term infants. Dev. Med. Child Neurol. 34, 507-515. https://doi.org/10.1111/j.1469-8749.1992.tb11471.x.

Taylor, M.J., Saliba, E., Laugier, J., 1996. Use of evoked potentials in preterm neonates. Arch. Dis. Child. Fetal Neonatal Ed. 74 (1), F70-F76. https://doi.org/10.1136/ fn.74.1.F70.

Toda, Tomohisa, Homma, Daigo, Tokuoka, Hirofumi, Hayakawa, Itaru, Sugimoto, Yukihiko, Ichinose, Hiroshi, Kawasaki, Hiroshi, 2013. Birth regulates the initiation of sensory map formation through serotonin signaling. Dev. Cell 27 (1), 32-46. https://doi.org/10.1016/j.devcel.2013.09.002.

Tombini, M., Pasqualetti, P., Rizzo, C., Zappasodi, F., Dinatale, A., Seminara, M., Ercolani, M., Rossini, P.M., Agostino, R., 2009. Extrauterine maturation of somatosensory pathways in preterm infants: A somatosensory evoked potential study. Clin. Neurophysiol. 120 (4), 783-789. https://doi.org/10.1016/j. clinph.2008.12.032.

Tsuneishi, S., Casaer, P., 2000. Effects of preterm extrauterine visual experience on the development of the human visual system: a flash VEP study. Dev. Med. Child Neurol. 42, 663-668. https://doi.org/10.1017/s0012162200001225.

Vaart, Marianne, Duff, Eugene, Raafat, Nader, Rogers, Richard, Hartley, Caroline, Slater, Rebeccah, 2019. Multimodal pain assessment improves discrimination between noxious and non-noxious stimuli in infants. Paediatr. Neonatal Pain 1 (1), 21-30. https://doi.org/10.1002/pne2.v1.110.1002/pne2.12007.

Van Braeckel, K.N.J.A., Taylor, H.G., 2013. Visuospatial and visuomotor deficits in preterm children: the involvement of cerebellar dysfunctioning. Dev. Med. Child Neurol. 55 (Suppl 4), 19-22. https://doi.org/10.1111/dmcn.12301.

Vanderah, T., Gould, D.J., 2020. Nolte's The Human Brain: An Introduction to its Functional Anatomy, 8th ed. Elsevier, Philadelphia.

Vanhatalo, Sampsa, Jousmäki, Veikko, Andersson, Sture, Metsäranta, Marjo, 2009. An easy and practical method for routine, bedside testing of somatosensory systems in extremely low birth weight infants. Pediatr. Res. 66 (6), 710-713. https://doi.org/ 10.1203/PDR.0b013e3181be9d66.

Walker, S.M., Franck, L.S., Fitzgerald, M., Myles, J., Stocks, J., Marlow, N., 2009. Longterm impact of neonatal intensive care and surgery on somatosensory perception in 
children born extremely preterm. Pain 141, 79-87. https://doi.org/10.1016/j. pain.2008.10.012.

Wallois, F., 2010. Synopsis of maturation of specific features in EEG of premature neonates. Neurophysiologie Clinique/Clin. Neurophysiol. 40 (2), 125-126. https:// doi.org/10.1016/j.neucli.2010.02.001.

Wallois, F., Routier, L., Bourel-Ponchel, E., 2020. Impact of prematurity on neurodevelopment, in: Gallagher, A., Bulteau, C., Cohen, D., Michaud, J.L. (Eds.), Handbook of Clinical Neurology, Neurocognitive Development: Normative Development. Elsevier, pp. 341-375. https://doi.org/10.1016/B978-0-444-641502.00026-5.

Welch, Martha G., Myers, Michael M., Grieve, Philip G., Isler, Joseph R., Fifer, William P., Sahni, Rakesh, Hofer, Myron A., Austin, Judy, Ludwig, Robert J., Stark, Raymond I., 2014. Electroencephalographic activity of preterm infants is increased by Family Nurture Intervention: A randomized controlled trial in the NICU. Clin. Neurophysiol. 125 (4), 675-684. https://doi.org/10.1016/j.clinph.2013.08.021.

Wess, Jessica M., Isaiah, Amal, Watkins, Paul V., Kanold, Patrick O., 2017. Subplate neurons are the first cortical neurons to respond to sensory stimuli. Proc. Natl. Acad. Sci. U. S. A. 114 (47), 12602-12607. https://doi.org/10.1073/pnas.1710793114.

Whitehead, K., Papadelis, C., Laudiano-Dray, M.P., Meek, J., Fabrizi, L., 2019. The Emergence of Hierarchical Somatosensory Processing in Late Prematurity. Cereb. Cortex 29, 2245-2260. https://doi.org/10.1093/cercor/bhz030.
Whyte, Hilary E.A., 1993. Visual-evoked potentials in neonates following asphyxia. Clin. Perinatol. 20 (2), 451-461.

Whyte, H.E., Pearce, J.M., Taylor, M.J., 1987. Changes in the VEP in preterm neonates with arousal states, as assessed by EEG monitoring. Electroencephalogr. Clin. Neurophysiol./Evoked Potentials Section 68 (3), 223-225. https://doi.org/10.1016/ 0168-5597(87)90030-X.

Wickremasinghe, A.C., Rogers, E.E., Johnson, B.C., Shen, A., Barkovich, A.J., Marco, E.J., 2013. Children born prematurely have atypical sensory profiles. J. Perinatol. 33 (8), 631-635. https://doi.org/10.1038/jp.2013.12.

Woody, Charles D., 1967. Characterization of an adaptive filter for the analysis of variable latency neuroelectric signals. Med. Biol. Eng. 5 (6), 539-554. https://doi org/10.1007/BF02474247.

Worley, A., Fabrizi, L., Boyd, S., Slater, R., 2012. Multi-modal pain measurements in infants. J. Neurosci. Methods 205 (2), 252-257. https://doi.org/10.1016/j. jneumeth.2012.01.009.

Zimmerman, E., Lahav, A., 2013. Ototoxicity in preterm infants: effects of genetics, aminoglycosides, and loud environmental noise. J. Perinatol. 33 (1), 3-8. https:// doi.org/10.1038/jp.2012.105. 